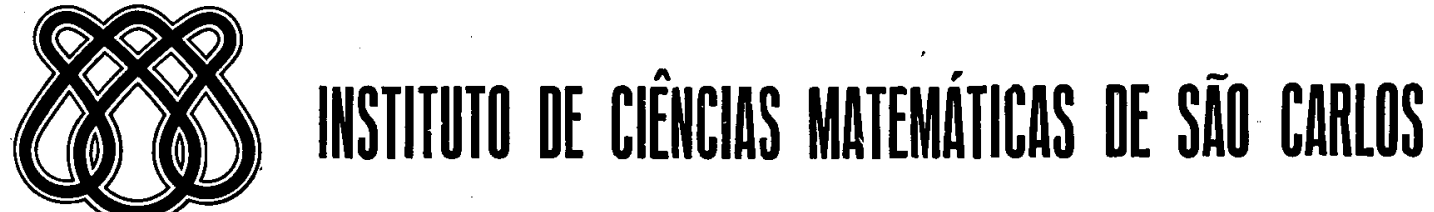 \\ I.C.M.S.C.

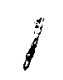

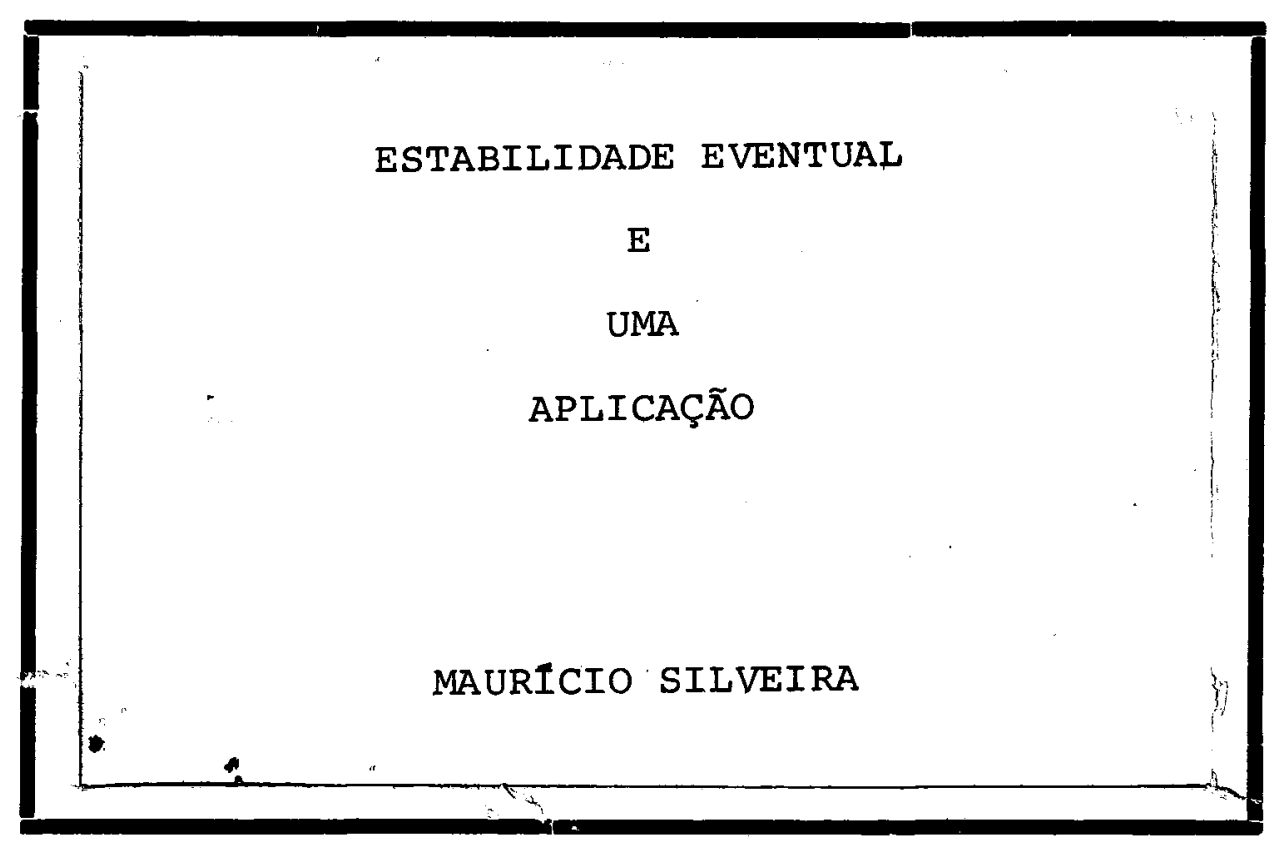

UNIVERSIDADE DE SÃO PAULO

SÃO CARLOS - SÃO PAULO

BRASIL 


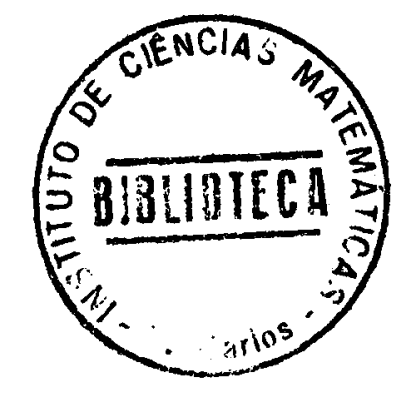

ESTABILIDADE EVENTUAL

E

UMA

APLICAÇÃO

MAURICIO SILVEIRA

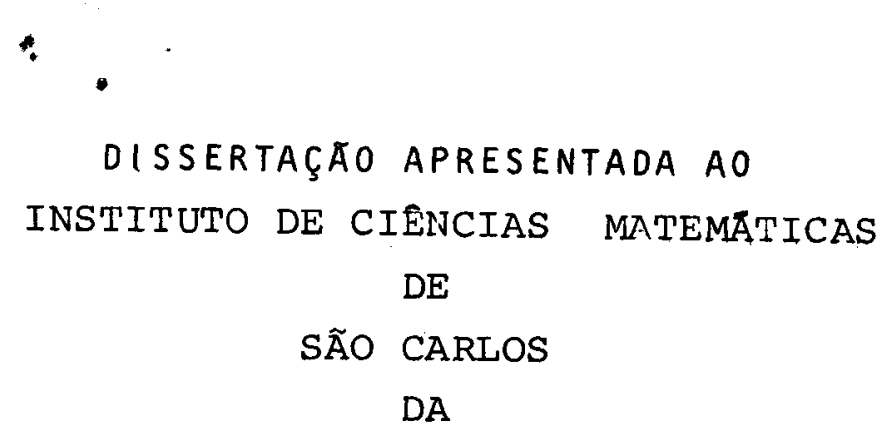

UNIVERSIDADE DE SÃO PAULO

PARA OBTENÇÃo DO TITULO dE MESTRE

EM

MATEMATICA

ORIENTADOR:

PROF. DR. NELSON ONUCHIC 


\title{
ESTABILIDADE EVENTUAL
}

E UMA

APLICAÇÃO

\author{
MAURÍCIO SILUEIRA \\ UNIVERSIDADE FEDERAL DE SATO CARLOS \\ DEPARTAMENTO DE MATEMATICA
}

ORIENTADOR: PROF. DR. NELSON ONUCHIC

INSTITUTO DE CIENCIAS MATEMATICAS

DE SÃO CARLOS - USP 
"O Universo estā sempre presente a nōs, com todas as suas forças e grandezas; para o percebermos, uma só coisa $\bar{e}$ necessāria: crearmos em

nōs suficientes receptividades para sintonizarmos a nassa antena individual com as ondas da emissora universal".

M. Gandhi 
Os agradecimentos iniciais são dirigidos às seguintes pessoas :

Dr. NELSON ONUCHIC - cuja orientação segura, disponi bilidade impar, relevante acolhimento; fizeram com que este trabalho chegasse à sua plena realização, bem como foram fato res integrantes na minha formação profissional e humana.

Dr. JANEY ANTONIO DACCACH - pelo exemplo constante de sobriedade profissional, e estímulo dado desde os instantes ini ciais na Universidade.

Dr. GILBERTO FRANCISCO LOIBEL - por ter-me dispensado atenção de um verdadeiro "gentleman" por ocasião de minha transferência do IME ao ICMSC.

Dra. ELZA FURTADO GOMIDE - pelo apoio que me foi dis pensado nos momentos iniciais vividos junto ao Instituto de Ma temática e Estatística da Universidade de são Paulo.

Dr. nATALINO ADELMO DE MOLFETTA - amigo constante, que sempre me deu o apoio e confiança necessários, possibilí tando a elaboração do meu programa de mestrado em breve tempo.

Dr. ORLANDO F. LOPES - pelos momentos de completa dis ponibilidade na discussão de alguns tópicos referentes a este trabalho.

Agradeço de forma especial aos colegas: LUIZ CARLOS PAVLU, e ADALBERTO SPEZAMIGLIO, que com valiosas críticas e oportunas sugestões, participaram construtivamente na elabora ção destas notas. 
Agradeço ainda: a todos os funcionários da gráfica da Universidade Federal de São Carlos, pelo acurado trabalho de impressão, e à Srta. MARIA JOSE GUALTIERI pelo esmero e a dedi cação com a qual datilografou esta dissertação.

Enfim a todos os colegas do ICMSC e da UFSCar, pelo ambiente de trabalho entusiástico e acolhedor sempre encontra do.

Este trabalho só foi possível graças ao apoio das entidades CAPES, CNPq, FAPESP, FINEP. 
A B S T R A C T

The main objective of this work is to study certain types of stability of the origin relatively to one system of Ordinary Differential Equations. We observe that the origin need not to be an equilibrium point of the system under consi deration.

Such types of stability that may play an important ro le in the theory of adaptive control systems, are known as eventual stability.

The concept of eventual stability for systems of or dinary differential equations is a natural extension of the concept of stability in the sense of Lyapunov.

We present definitions, basic facts and qualitative properties of the solutions of the menctioned system.

We improve results and examples discussed by J.P. La Salle, R.J. Rath and V. Lakshmikanthan.

The techniques used in this dissertation are provided by the ideas contained in the theory of Lyapunov functions. 
CAPITULO 0 - APRESENTAÇÃO

CAPITULO $1-$ ESTABILIDADE EVENTUAL .............. 1

$1.1-$ Introdução $\ldots \ldots \ldots \ldots \ldots \ldots \ldots \ldots \ldots \ldots$

$1.2-$ Definições $\ldots \ldots \ldots \ldots \ldots \ldots \ldots \ldots \ldots \ldots \ldots \ldots$

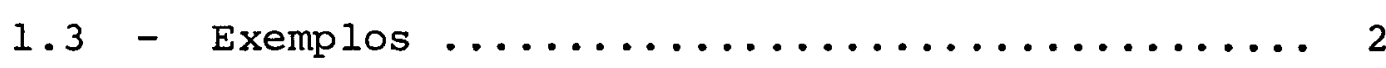

CAPITULO 2 - ALGUMAS PROPRIEDADES ............... 9

CAPITULO 3 - RELAÇÕES COM A TEORIA DE LIAPUNOFF ....... 19

3.1. - Estabilidade Eventual Uniforme ......... 20

3.2 - Estabilidade Eventual Assintótico-Uniforme ...................... 23

CAPITULO 4 - REGIÕES DE ATRAÇÃO - EM ESTABILIDADE EVEN TUAL ASSINTOTICA ................ 25

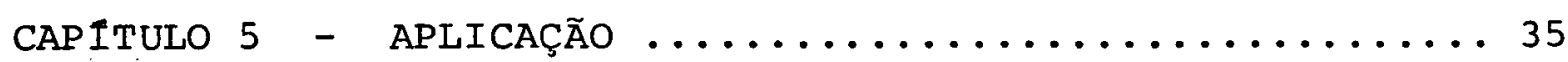

5.1 - Introdução à Teoria do Controle ......... 35

5.2 - Um Problema de Estabilidade em Controle ... 38

APENDICE 
CAPITULO 0

\section{APRESENTAÇÃO}

Em vārias situações concretas poderemos ser levados a considerar a estabilidade de estados que não são de equilí brio.

Tal comportamento pode não se enquadrar dentro da teo ria de estabilidade proposta por Liapunoff. Aliās; a condição "ser estável" em se tratando de estabilidade da origem, está associada intrinsecamente, a exigência de que esta seja um ponto de equilibrio.

Os tipos de estabilidade a serem abordados nestas no tas, são de estabilidades de estados não necessariamente de equilíbrio, mas que com o passar do tempo, tendem a se compor tar "sempre mais" como estados de equilỉbrio estāvel, ou mesmo assintoticamente estável.

Determinado tipo de estabilidade encontra um campo de aplicação imediata na teoria de sistemas de controle adaptạ veis.

Se o problema de controle for considerado com "rea lismo", então este é o tipo de estabilidade que esperamos en contrar.

$\mathrm{Na}$ parte inicial deste trabalho são dados os concei tos de estabilidade eventual.

Posteriormente algumas propriedades bāsicas deste tị po de estabilidade são obtidas.

Aspectos comparativos com a teoria de Liapunoff sobe 
jamente conhecida são tambēm salientados.

Como complementação apresentamos teoremas que nos dão informações da teoria qualitativa relacionada ao campo de estą bilidade eventual. Por exemplo, pode-se dar uma estimativa re ferente a uma região de estabilidade eventual.

A parte conclusiva traz um exemplo de como a teoria pode ser aplicada.

Esta dissertação é fortemente motivada numa publica ção de J.P. LASALLE e R.J. RATh. 


\section{CAPITULO 1}

\section{ESTABILIDADE EVENTUAL}

\section{1 - INTRODUÇAO}

Consideremos o sistema de equações diferenciais ordí nārias :

$$
\dot{x}=f(t, x)
$$

onde $f(t, x)$ é contínua, e satisfaz alguma condição de unicida de em relação aos dados iniciais, no conjunto $\Omega \mathrm{C} I R^{n+1}$ defini da por $\Omega=\{(t, x) \mid t \geq 0$ e ||$x||<\rho \leq \infty\}$.

Aqui ||$x||$ é a norma euclideana do $I^{n}$ dada por

$$
\| x||=\left(\sum_{i=1}^{n} x_{i}^{2}\right)^{1 / 2}
$$

onde $x=\left(x_{1}, x_{2}, \ldots, x_{n}\right)$ é um vetor qualquer do $\mathbb{R}^{n}$.

Denotamos por $x\left(t, t_{0}, x_{0}\right)$ a solução de (I) tal que $x\left(t_{0}, t_{0}, x_{0}\right)=x_{0}$. Considederamos a solução $x\left(t, t_{0}, x_{0}\right)$ estendida ao seu máximo intervalo de definição aberto à direita.

E bem conhecido que dentro das hipōteses dadas acima, as soluções de (I) são continnuas em relação às condições ini ciais.

Se $J=[0, \infty)$, dizer que $f \in C\left[J \times I R^{n}, I R^{n}\right]$ equivale a dizer que $f \bar{e}$ contínua no par $(t, x) \forall t \in J, \forall x \in \mathbb{R}^{n}$.

\section{2 - DEFINIÇOES}

0.1 - A origem $x=0$ è dita eventualmente uniformemente es tāvel se para cada $\varepsilon>0$ existem números positivos 
$\delta=\delta(\varepsilon)$ e $T=T(\varepsilon)$ de modo que se ||$x_{0}||<\delta$, então ||$x\left(t, t_{0}, x_{0}\right)||<\varepsilon \quad \forall t$ tal que $t \geq t_{0} \geq T$.

D.2 - A origem $x=0$ è dita eventualmente quase uniformemen te assintoticamente estāuel se para cada $\varepsilon>0$ exis tem nümeros positivos $\delta_{0}, T_{0}$ e $T=T(\varepsilon)$ tal que; se ||$x_{0}|| \leq \delta_{0}$, então ||$x\left(t, t_{0}, x_{0}||<\varepsilon \quad \forall t\right.$ tal que $t \geq$ $\geq t_{0}+T \operatorname{com} t_{0} \geq T_{0}$

D.3 - A origem $x=0$ é dita eventualmente uniformemente as sintoticamente estāuel se ela satisfaz 0.1 e 0.2 si multaneamente.

D.4 - A origem $x=0$ é dita eventualmente exponencialmente assintoticamente estāvel se existem constantes $L>0$ e $\alpha>0$ tais que ||$x\left(t, t_{0}, x_{0}\right)||<L|| x_{0} \| e^{-\alpha\left(t-t_{0}\right)} t \geq t_{0}$, com $0<r<|| x_{0}||<\rho$ e $t_{0} \geq \theta(r)$ onde $\theta(r)$ è uma função decrescente de $r$ no intervalo $(0, \rho)$.

Neste parágrafo, bem como no parágrafo precedente; $D_{i}$ e $E_{i} ; \quad i=1,2,3,4$ se referem às definições e exemplos dos dí ferentes tipos de estabilidade eventual para o sistema de equa ções diferenciais ordinārias (I) .

\section{$1.3-$ EXEMPLOS}

No desenvolver deste parágrafo, faremos referência al 
gumas vezes, ao espaço

$$
\begin{aligned}
M_{0}= & \left\{\psi \in C\left([0, \infty), I R^{n}\right) \mid \int_{t}^{t+1}\|\psi(s)\| \text { ds } \rightarrow 0\right. \text { quando } \\
& t \rightarrow \infty\} .
\end{aligned}
$$

o seguinte lema é de interesse para o que fa remos adiante.

LEMA :

$$
M_{1}=\left\{\psi \in C\left([0, \infty), R_{+}\right) \mid \int_{t}^{t+1} \psi(s) d s \rightarrow 0 \text { quando } t \rightarrow\right.
$$

$\rightarrow \infty\}$, então:

$$
\psi \in M_{1} \Rightarrow \lim _{t \rightarrow \infty} e^{-\sigma t} \int_{1}^{t} e^{\sigma s} \psi(s) d s=0
$$

para qualquer $\sigma>0$.

Para uma demonstração consultar uma das referências (6) ou (8).

E.1 - Consideremos o sistema (I) reduzido ao caso escalar

$$
\dot{x}=-a x+\psi(t) \text { onde } \begin{aligned}
& \| a>0 \\
& \| \psi \in M_{0}(n=1)
\end{aligned}
$$

Logo, a origem $x=0$ de (II) satisfaz a definição 0.1 . De fato, dado $\varepsilon>0$, para cada escolha de $t_{0} \geq 0$ te mos :

$$
x\left(t, t_{0}, x_{0}\right)=e^{-a\left(t-t_{0}\right)} x_{0}+e^{-a t} \int_{t_{0}}^{t} e^{a s} \psi(s) d s(1.3 .1)
$$

Agora como $t \geq t_{0} \rightarrow t-t_{0} \geq 0 \rightarrow-a\left(t-t_{0}\right) \leq 0$; decor re então que $e^{-a\left(t-t_{0}\right)} \leq 1$. Por conseguinte, se escolhermos $\delta=\frac{\varepsilon}{2}$ a primeira parcela de (1.3.1) é majorada por: 


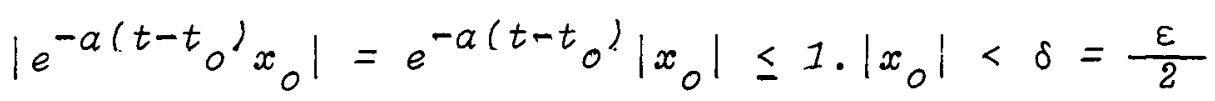

De acordo com o lema

$\psi \in M_{0} \rightarrow \lim _{t \rightarrow \infty} e^{-a t} \int_{1}^{t} e^{a s}|\psi(s)| d s=0$.

Portanto, existe:

$T=T(\varepsilon)\left|e^{-a t} \int_{1}^{t} e^{a s}\right| \psi(s) \mid d s<\frac{\varepsilon}{2}$ para $t \geq T \geq 1$.

Logo, dado $\varepsilon>0, \exists \delta=\frac{\varepsilon}{2}$ e $T=T(\varepsilon) \geq 1$ tal que $\forall t \geq t_{o} \geq T$ temos:

$$
\begin{aligned}
& \left|x\left(t, t_{0}, x_{0}\right)\right|=\left|e^{-a\left(t-t_{0}\right)} x_{0}+e^{-a t} \int_{t_{0}}^{t} e^{a s} \psi(s) d s\right| \leq
\end{aligned}
$$

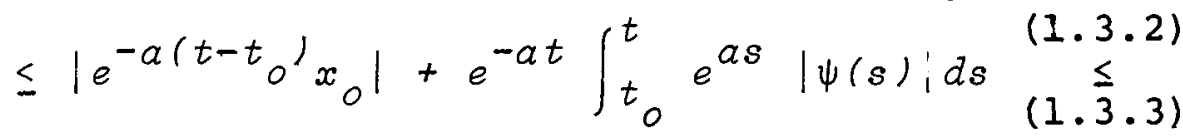

$$
\begin{aligned}
& \leq \frac{\varepsilon}{2}+\frac{\varepsilon}{2}=\varepsilon \text {. }
\end{aligned}
$$

Observe que $\psi \in M_{0} \rightarrow|\psi| \in M_{1}$, então

$\int_{t_{0}}^{t} e^{a s}|\psi(s)| d s \leq \int_{1}^{t} e^{a s}|\psi(s)| d s$

para $t \geq t_{0} \geq T \geq 1$

Mais adiante veremos um caso mais geral de estabilida de eventual que tem como caso particular o E.1. Por ora nos detemos simplesmente nas seguintes observações:

1) No caso em que $\psi \neq 0$ o exemplo dado mostra que es tabilidade uniformemente eventual não requer necessariamente que a origem seja ponto de equilíbrio.

2) Em E.1 impondo as condições $\psi \notin M_{0}$ e $\psi \geq 0$ já não podemos concluir 0.1 para a origem do sistema (II).

De fato:

$\psi \notin M_{0}(n=1) \rightarrow \exists \alpha>0$ 
e uma sequência $t_{n} \rightarrow \infty \operatorname{com} n \rightarrow \infty \mid \int_{t_{n}}^{t} \psi(s) d s \geq \alpha \cdot \forall n$. Logo para cada escolha de $t_{0} \geq 0$ e $x_{0} \geq 0$ temos:

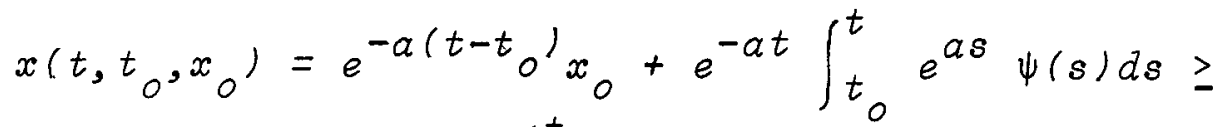

$$
\begin{aligned}
& \geq e^{-a t} \int_{t_{0}}^{t} e^{a s} \psi(s) d s
\end{aligned}
$$

Portanto para todo $n$ suficientemente grande temos:

$$
x\left(t_{n}+1, t_{0}, x_{0}\right) \geq e^{-a\left(t_{n}+1\right)} \int_{t_{0}^{t} n^{+1}}^{e^{a s}} x(s) d s \geq e^{-\alpha} \alpha \text {. }
$$

Logo para $0<\varepsilon<e^{-a} \alpha$ a origem $x=0$ do sistema (II)

não satisfaz D.l.

o exemplo seguinte para o caso em que $n=1$ coincide com E.1. Neste exemplo a origem $x=0$ satisfaz às exigências das definições D.1, D.2 e D.3. O caso $\psi(t) \neq 0$ mostra que a origem pode satisfazer tais condições sem ser um ponto de equi líbrio para o sistema.

E.2 - Consideremos o sistema

$$
\dot{x}=A x+\psi(t)
$$

onde $A \in M(I R, n \times n), \psi(t)=\left(\psi_{1}(t), \psi_{2}(t), \ldots, \psi_{n}(t)\right) \in I^{n}$ qual quer que seja $t \in[0, \infty)$ com $\psi$ contínua em $I R+, \psi \in M_{0}$.

Vamos ainda adicionar a exigência que a matriz $A$ do sistema dado admita somente valores característicos com parte real negativa.

Nestas condições a origem $x=0$ do sistema (II) satis faz D.1.

De fato, a solução $x(t)=x\left(t, t_{0}, x_{0}\right)$ do sistema è dạ da por

$$
x(t)=e^{A\left(t-t_{0}\right)} x_{0}+\int_{t_{0}}^{t} e^{A(t-s)} \psi(s) d s .
$$


Agora a condição (1.3.4) garante a existência de cons tantes $K>0$ e $\alpha>0$ com $0<\alpha<-R_{e}\left(\alpha_{J}\right), J=1,2, \ldots, n_{i}$ tal que ||$e^{t A}|| \leq K e^{-\alpha t} t \geq 0$. Portanto;

$$
|| e^{A\left(t-t_{0}\right)}|| \leq K \cdot e^{-\alpha\left(t-t_{0}\right)} t \geq t_{0}
$$

Porèm

$$
\begin{aligned}
& t \geq t_{0} \mathrm{e} \alpha>0 \rightarrow \alpha\left(t-t_{0}\right) \geq 0 \rightarrow-\alpha\left(t-t_{0}\right) \leq 0 \rightarrow \\
& \rightarrow e^{-\alpha\left(t-t_{0}\right)} \leq 1 \rightarrow K e^{-\alpha\left(t-t_{0}\right)} \leq K
\end{aligned}
$$

Portanto dado $\delta=\frac{\varepsilon}{2 K}$; se $\left\|x_{0}\right\|<\delta$ a norma da pri meira parcela de (1.3.5) é majorada por $\frac{\varepsilon}{2}$. De fato:

$$
\begin{aligned}
& || e^{-A\left(t-t_{0}^{\prime} x_{0}\right.}|\leq|\left|e^{-A\left(t-t_{0}\right)}\right||| \mid x_{0} \| \leq \\
& \leq K e^{-\alpha\left(t-t_{0}\right)}|| x_{0}|| \leq K .|| x_{0}|| \leq K . \delta=\frac{\varepsilon}{2}
\end{aligned}
$$

$$
1.3 .7
$$

Entretanto a condição $\psi \in M_{0}$ conjuntamente com o lema antes mencionado garantem a existência de

$$
\begin{aligned}
T= & T(\varepsilon) \geq 1\left|e^{-\alpha t} \int_{1}^{t} e^{\alpha s}\right||\psi(s)| \mid d s<\frac{\varepsilon}{2 K} \forall t \geq \\
& \geq T \geq 1
\end{aligned}
$$

Desta forma a segunda parcela da expressão (1.3.5) é majorada em norma por:

$$
\begin{aligned}
& \left\|\int_{t_{0}}^{t} e^{A(t-s)} \psi(s) d s|| \leq \int_{t_{0}}^{t}\right\| e^{A(t-s)} \psi(s)\left\|d s \leq \int_{t_{0}}^{t}\right\| e^{A(t-s)} \| \text {. } \\
& || \psi(s)\left\|d s \leq k \int_{t_{0}}^{t} e^{-\alpha(t-s)}|| \psi(s)\right\| d s=K e^{-\alpha t} \int_{t_{0}}^{t} e^{\alpha s} \\
& || \psi(s)|| d s \leq K e^{-\alpha t} \int_{1}^{t} e^{\alpha s}|| \psi(s) \| d s \underset{1.3 .9}{<} K \cdot \frac{\varepsilon}{2 K}=\frac{\varepsilon}{2} \text { se } \\
& t \geq t_{0} \geq T \geq 1 \\
& \text { o uso da desigualdade triangular na relação (1.3.5); }
\end{aligned}
$$
levando em conta as expressões $(1.3 .8)$ e $(1.3 .10)$ fornecem di 
retamente o resultado desejado.

Vale salientar que se $T_{0}=1$ e $\delta_{0}$ é um real qualquer fixado tal que $0<\delta_{0}<\delta$, 0 E.2 está nas condições da defini ção D. 3 .

E.3 - Este exemplo se enquadra dentro das exigências da defini Ção D. 4 .

Consideremos o sistema dado por $\dot{x}=-a x+b(t)$ onde $\alpha>0$ e $b$ é definida como segue:

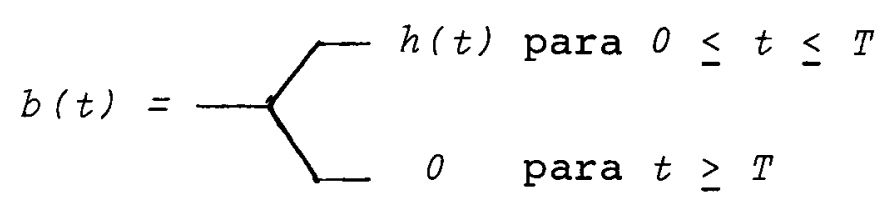

$h$ sendo contínua no intervalo $[0, \infty)$ satisfazendo $h(T)=0$ com $T>0$.

A definição D.4 fica automäticamente verificada.

De fato; se tomarmos $\theta(r)=\frac{T}{r}$ a condição $t_{0} \geq \theta(r)$ fornece $t_{0} \geq \frac{T}{r}>T$ se $\rho=1$ pois:

$$
r<|| x_{0}||<\rho=1 \rightarrow \frac{1}{r}>1 \rightarrow \frac{T}{r}>T .
$$

Desta forma a solução do sistema dado para $t_{0} \geq T$ é dada por $x(t)=e^{-\alpha\left(t-t_{0}{ }^{\prime} x_{0}\right.}$. Logo D.4 está verificada se es colhermos $L=1$ e $x_{0}=x\left(t_{0}\right)$ com $0<r<|| x_{0}||<1$.

Tal exemplo pode ser facilmente estendido ao $\mathbb{I R}^{n}$ toman do-se $\dot{x}=A x+\psi(t)$ onde $A \in M(I R, n \times n)$ admite autovalores com parte real negativa e $\psi(t)=\left(\psi_{1}(t), \psi_{2}(t), \ldots, \psi_{n}(t)\right)$ è uma função contínua tal que existe $T>0 \operatorname{com} \psi_{i}(t)=0 \forall t \geq T$ e $\forall i \in\{1,2, \ldots, n\}$.

A verificação deste caso contém essencialmente a mes ma linha de raciocínio usada ao se estender o exemplo E.l ao exemplo E.2. 
CAP ITULO 2

\section{ALGUMAS PROPRIEDADES}

Este capitulo traz essencialmente algumas proprieda des bāsicas cos sistemas eventualmente estáveis.

O último teorema trata de estabilidade do tipo "even tual assintótico - uniforme" onde o sistema (I) é alterado por uma determinada perturbação, a qual nos è mencionada uma certa propriedade.

Faremos referência neste teorema a um resultado que é abordado em detalhes no apêndice destas notas.

A definição D.l nos diz que com o passar do tempo a origem tende a "atuar" sempre mais como um estado de equilibrio estāve1.

A estabilidade eventual tem um outro sentido intuití vo. Se a origem do sistema satisfaz D.l logo o sistema tem a propriedade que: se ele operou corretamente durante um período de tempo suficientemente grande, pode-se esperar que ele conti nue a operar adequadamente no futuro.

Esta interpretação de estabilidade eventual nos è dạ da pelo:

TEOREMA 2.1:

A origem $x=0$ satisfaz D.l se e somente se: dado $\varepsilon>0$, existem $\delta=\delta(\varepsilon)>0$ e $T=T(\varepsilon)>0$ tais que se $\left\|x\left(t_{1}, t_{0}, x_{0}\right)\right\|<$ $<\delta$ para algum $t_{1} \geq T$, então ||$x\left(t, t_{0}, x_{0}\right)_{i} \mid<\varepsilon \forall t \geq t_{1}$. D. - Para efeito de simplificação a notação $x_{1}$ indicará o 
valor $x\left(t_{1}, t_{0}, x_{0}\right)$. Observamos que aquilo que o teorema propõe è algo do tipo "independência do $t_{0}$ fixado". No sentido que: se $t_{1}$ e $x_{1}$ são os valores que verificam a propriedade proposta pelo teorema, estes passam a ser os novos valores de $t_{0} e x_{0}$ da definição D. 1 .

$+\operatorname{Seja} x_{0} \in I^{n}$ satisfazendo ||$x_{0}||<\delta$, e considere mos $x\left(t, t_{0}, x_{0}\right)$ a solução de (I) com $t_{0} \geq T$.

Como existe uma certa arbitrariedade na escolha de $t_{1}$ do Teorema (2.1), tomando $t_{1}=t_{0}$ temos:

$$
|| x_{1}||=|| x_{0}||<\delta \rightarrow|| x\left(t, t_{0}, x_{0}\right) \mid<\varepsilon \quad \forall t \geq t_{1}=t_{0} \geq T ;
$$

portanto $x=0$ satisfaz D.1.

$\rightarrow x=0$ satisfaz D.I $\rightarrow$ dado $\varepsilon>0, \exists \delta=\delta(\varepsilon)$ e $T=$ $=T(\varepsilon)$ nümeros positivos tais que

$$
|| x_{0}||<\delta \rightarrow|| x\left(t, t_{0}, x_{0}\right)||<\varepsilon \forall t \geq t_{0} \geq T
$$

Vamos supor em adição que para algum $t_{1} \geq T$ temos ||$x_{1} i \mid<\delta$. Temos desta forma dois casos a considerar:

$$
\begin{aligned}
& \mid: \text { a) } t_{1} \geq t_{0} \geq T \\
& i \text { b) } t_{0} \geq t_{1} \geq T
\end{aligned}
$$

- ocorrendo (a) temos:

$$
\forall t\left|t \geq t_{1} \rightarrow t \geq t_{0} \stackrel{2.1 .1}{\rightarrow}\right|\left|x\left(t, t_{0}, x_{0}\right)\right| \mid<\varepsilon \quad \forall t \geq t_{1}
$$

1- ocorrendo (b); consideramos a solução $y(t)$ de (I) satisfá zendo $y\left(t_{1}, t_{1}, x_{1}\right)=x_{1}$.

$$
\text { Como }|| x_{1}||=|| y\left(t_{1}\right)||<\delta \text {, a definição D.1 aplica }
$$
da a solução $y$ nos dá ||$y\left(t, t_{1}, x_{1}\right)||<\varepsilon$ para $t \geq t_{1} \geq T$.

$$
\text { Mas } y\left(t_{1}, t_{1}, x_{1}\right)=x_{1} \rightarrow y \equiv x \text { para todo }
$$$$
t \rightarrow|| x\left(t, t_{0}, x_{0}\right)||<\quad \forall t \geq t_{1} \geq T
$$ 
e a verificação se completa.

Descrevemos agora um teorema que possue um caso simi lar na teoria de estabilidade segundo Liapunov; antes, porém, necessitamos de uma:

Definição: Dizemos que $V(t, x)$ é assintoticamente não positiva na região $\Omega$ do $R^{n+1}$ se $V(t, x) \leq \phi(t)$ para todo $0 \leq:|x| \mid<\rho, \quad t \in J \in \phi \in M_{1}(E .1)$.

Definindo $\dot{v}(t, x)$ na forma habitual, ou seja: $\dot{v}(t, x)=\frac{\partial v}{\partial t}+\left(\operatorname{grad}_{x} v\right) \cdot f(t, x)=\frac{\partial v}{\partial t}+\sum_{i=1}^{n} \frac{\partial v}{\partial x_{i}} f_{i}(t, x) ;$ recorrendo à definição acima, pode-se verificar o próximo teo rema (vide apêndice).

TEOREMA 2.2:

Seja $v(t, x)$ definida positiva em $\Omega$ satisfazendo $v(t, x) \rightarrow 0$ quando ||$x|| \rightarrow 0$ uniformemente em $t$ para $t \geq 0$. Se $\dot{v}(t, x)$ é assintoticamente não positiva, então a origem do sis tema (I) satisfaz D.I.

D. - Supondo $x=0$ não eventualmente uniformemente estável $\rightarrow \exists \varepsilon>0 \mid \forall \delta>0, \forall T>0 \quad \exists x(t)$ solução de $I$ de modo que ||$x_{0}||<\delta$, mas ||$x\left(\bar{t}, t_{0}, x_{0} \| \geq \varepsilon\right.$ para algum $\bar{t} \geq t_{0} \geq T$. Como $x(t)$ é contínua $\rightarrow \exists V=V_{\alpha}(\bar{t}) \mathrm{C} I R, \alpha>0$, tal que $\forall t \in V$ temos ||$x(t) \| \geq \frac{\varepsilon}{2} \cdot(2.2 .1)$ Agora como $v(t, x)$ é definida positiva $\rightarrow$ dado $\frac{\varepsilon}{2}>0, \exists n=n(\varepsilon)>0$ tal que $\frac{\varepsilon}{2} \leq|| x||<\rho$ implica $v(t, x) \geq n . \quad(2.2 .2)$

Ainda, se $v(t, x) \rightarrow 0$ quando ||$x|| \rightarrow 0$ uniformemente em $t \rightarrow$ dado $\frac{n}{2}>0, \exists \delta=\delta(\varepsilon)>0$ tal que se ||$x||<\delta \rightarrow$ 
$\rightarrow v(t, x)<\frac{n}{2} \cdot(2.2 .3)$

Logo, tomando $t=t_{0}$ a hipōtese nos dá

$$
\left\|x_{0}\right\|=\left\|x\left(t_{0}\right)\right\|<\delta \stackrel{2.2 .3}{\rightarrow} v_{0}=v\left(t_{0}, x_{0}\right)<\frac{n}{2}
$$

Portanto, integrando $v$ de $t_{0}$ a $\overline{\bar{t}}$ obtemos:

$v\left(\bar{t}, x\left(\bar{t}, t_{0}, x_{0}\right)\right)=v_{0}+\int_{t_{0}}^{\bar{t}} \dot{v}(s, x(s)) d s \rightarrow n \leq v_{0}+$

$+\int_{t_{0}}^{\bar{t}} \dot{v}(s, x(s)) d s \stackrel{2.2 .4}{\rightarrow} \int_{t_{0}}^{\bar{t}} \dot{v}(s, x(s)) d s>\frac{n}{2}>0$

Por hipōtese, $\dot{v}(t, x)$ é assintoticamente não positiva

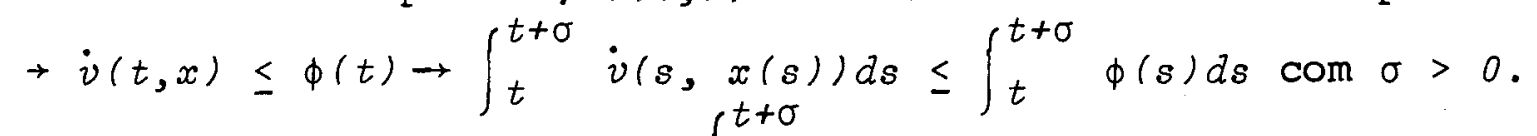

Dado que $\phi \in M_{1} \rightarrow \int_{t}^{t+\sigma} \phi(s) d s \rightarrow 0$ quando $t \rightarrow \infty$ para cada $\sigma>0 \rightarrow \int_{t}^{t+\sigma} \dot{v}(s, x(s)) d s \rightarrow 0$ quando $t \rightarrow \infty$.

Logo, $\exists T_{0}>0$, tal que $\forall t \geq T_{0}$ temos

$$
\int_{t}^{t+\sigma} \dot{v}(s, x(s)) d s<\frac{n}{2}
$$

Como na negação inicial temos $\forall T$, fazendo $T=T_{0^{\prime}}$ as expressões $(2.2 .5)$ e (2.2.6) nos fornecem uma contradição; e a prova se completa.

Aquilo que pretendemos agora é obter um análogo do Teorema (2.1) para o caso de estabilidade eventual assintótico - uniforme da origem.

Visando este objetivo a definição D. 3 pode ser refor mulada do seguinte modo:

D.'3 - A origem $x=0$ é dita eventualmente uniformemente as sintoticamente estāvel se:
i) satisfaz D.I;
ii) existe um $r>0$ e um $T_{0}$ tal que ||$x_{0}||<r$ e 


$$
t_{0} \geq T_{0} \text { implicam } x\left(t, t_{0}, x_{0}\right) \rightarrow 0 \text { quando } t \rightarrow \infty \text {. }
$$

A condição (ii) estabelece que, se depois de um tempo suficientemente longo, o sistema (I) se mantém perto da origem, então ele tenderā a origem com tendendo ao infinito. Nesta linha de idéias se encaixa o:

\section{TEOREMA 2.3:}

A condição (ii) da definição D'. 3 é equivalente a:Exis te $r>0$ e $T_{0}$ tal que ||$x\left(t_{1}, t_{0}, x_{0}\right) \|<r$ para algum $t_{1}>T_{0}$ então $x\left(t, t_{0}, x_{0}\right) \rightarrow 0$ quando $t \rightarrow \infty$.

D. - $\rightarrow$ Por hipótese, existe $r$ e $T_{0}$ tal que ||$x_{0}||<r$ e $t_{0} \geq T_{0}$ implicam $x\left(t, t_{0}, x_{0}\right) \rightarrow 0$ quando $t \rightarrow \infty$.

Em adição consideremos a solução $y\left(t, t_{1}, y_{1}\right)$ "̈o sis tema (I) tal que $y_{1}=x\left(t_{1}, t_{0}, x_{0}\right)=x_{1}$ com $t_{1} \geq T_{0}$ nas condi ções da hipótese. Logo: ||$y_{1}||=|| x_{1}||<r \operatorname{com} t_{1} \geq T_{0}$. Des ta forma a condição (2.3.1) aplicada à solução $y\left(t, t_{1}, y_{1}\right)$ nos dâ $y\left(t, t_{1}, y_{1}\right) \rightarrow 0$ quando $t \rightarrow \infty$. Dado que para $t=t_{1}$ temos $y_{1}=y\left(t_{1}, t_{1}, y_{1}\right)=x_{1}=x\left(t_{1}, t_{0}, x_{0}\right) \rightarrow y \equiv x \rightarrow x\left(t, t_{0}, x_{0}\right) \rightarrow 0$ quando $t \rightarrow \infty$.

↔) Sejam $r$ e $T_{0}$ os elementos da hipótese. Assim se para algum $t_{1} \geq T_{0}$ vale ||$x_{1}||=|| x\left(t_{1}, t_{0}, x_{0} \| \mid<r\right.$, então $x\left(t, t_{0}, x_{0}\right) \rightarrow 0$ quando $t \rightarrow \infty$.

Tomemos $x_{0}$ um vetor qualquer do $I^{n}$ satisfazendo $\left\|x_{0}\right\|<r$ e consideremos a solução $x\left(t, t_{0}, x_{0}\right) \operatorname{com} t_{0} \geq T_{0}$. Pondo $t_{1}=t_{0}$, a condição (2.3.2) é claramente satisfeita. Decorre que $x\left(t, t_{0}, x_{0}\right) \rightarrow 0$ quando $t \rightarrow \infty$ e comple tamos a prova.

o próximo teorema possue um correspondente para o ca 
so de Estabilidade Eventual Uniforme dado pelo Teorema 2.2.

TEOREMA 2.4:

Seja $v(t, x)$ satisfazendo as condições:

a) $v(t, x)$ é definida positiva em $\Omega$;

b) $v(t, x) \rightarrow 0$ quando ||$x|| \rightarrow 0$ uniformemente em $t$, para $t \geq 0$

c) $\dot{v}(t, x) \leq-c(|x|)+h(t)$ em $J \times \Omega$ com

$$
\begin{aligned}
& ||-c \text { definida positiva em } \Omega \\
& \| \mid-h \in M_{1}
\end{aligned}
$$

Então a origem $x=0$ satisfaz D. 3 .

D. - De $\dot{v}(t, x) \leq-c(|x|)+h(t) \rightarrow \dot{v}(t, x) \leq h(t)$ com $h \epsilon$ Teor.

E $M_{1} \underset{2.2}{\rightarrow} x=0$ satisfaz D. $1 . \quad$ (2.4.1)

Agora como a função $v(t, x)$ satisfaz as condições $(a)$ e

(b) $\rightarrow$ existem funções $f$ e $g$ na classe $K$ tal que

$$
f(|x|) \leq v(t, x) \leq g(|x|)
$$

Como $f$ e $g \dot{\epsilon} K$, então existem $\rho_{1}$ e $\rho_{2}$ satisfazendo $0<\rho_{1}<$ $<\rho_{2}<\rho$ tal que $g\left(\rho_{1}\right)<f\left(\rho_{2}\right) \cdot \quad(2.4 .2)$

Desta forma, afirmamos que existe $T_{0}=T_{0}\left(\rho_{2}\right)$ tal que ||$x_{0}||<\rho_{1} \rightarrow|| x(t)||<\rho_{2} \quad \forall t \geq t_{0} \geq T_{0}$.

De fato; caso contrário, para qualquer $T_{0}, \quad 3 t_{1}, t_{2}$ $\operatorname{com} T_{0} \leq t_{0}<t_{1}<t_{2} \operatorname{com}|| x_{1}||=\rho_{1}, \quad|| x_{2}||=\rho_{2}$ e $\rho_{1}<$ ||$x(t)||<\rho_{2}$ para $t \in\left(t_{1}, t_{2}\right)$.

Aqui $x_{1}$ e $x_{2}$ indicam respectivamente os valores $x\left(t_{1}, t_{0}, x_{0}\right)$ e $x\left(t_{2}, t_{0}, x_{0}\right)$.

Logo: 


$$
v\left(t_{1}, x_{1}\right) \leq g\left(\left|x_{1}\right|\right)=g\left(\rho_{1}\right)<_{2.4 .2} f\left(\rho_{2}\right)=f\left(\left|x_{2}\right|\right) \leq v\left(t_{2}, x_{2}\right)
$$

e portanto:

$$
0<v\left(t_{2}, x_{2}\right)-v\left(t_{1}, x_{1}\right) \leq-\int_{t_{1}}^{t_{2}} c(|x(s)|) d s+\int_{t_{1}}^{t_{2}} h(s) d s .
$$

Mas :

$$
\rho_{1} \leq|| x|| \leq \rho_{2} \rightarrow c(|x|) \geq c\left(\rho_{1}\right)=\sigma>0 \rightarrow-c(|x|) \leq-\sigma<0
$$

e assim:

$0<v\left(t_{2}, x_{2}\right)-v\left(t_{1}, x_{1}\right) \leq-\sigma\left(t_{2}-t_{1}\right)+\int_{t_{1}}^{t} h(s) d s \rightarrow \int_{t_{1}}^{t_{2}} h(s) d s>$

$$
>\sigma\left(t_{2}-t_{1}\right)>0, \forall T_{0},
$$

com $t_{2}>t_{1}>t_{0} \geq T_{0} \circ$ que é um absurdo, pois $h \in M_{1}$.

Resta provar que $\left\|x_{0}\right\|<\rho_{1} \operatorname{com} t_{0} \geq T_{0}=T_{0}\left(\rho_{2}\right)$, en tão $x\left(t, t_{0}, x_{0}\right) \rightarrow 0 \operatorname{com} t \rightarrow \infty$.

De fato; de (2.4.1) $x=0$ satisfaz D.1; dado $\varepsilon>0$, existem $\delta=\delta(\varepsilon)$ e $T_{0}=T_{0}(\varepsilon)$ tal que

$$
|| x_{0}||<\delta \rightarrow|| x\left(t, t_{0}, x_{0}\right) \mid<\varepsilon
$$

para $t \geq t_{0} \geq T_{0} \cdot(2.4 .3)$

Sem perda de generalidades podemos assumir que $\delta<$ $<g^{-1}(f(\varepsilon))$ e vamos escolher $T_{0}=\max \left\{T_{0}\left(\rho_{2}\right), T_{0}(\varepsilon)\right\}$. Defini mos agora $T=T(\varepsilon)=\frac{g\left(\rho_{1}\right)}{c(\delta)}+\frac{\varepsilon}{\tilde{2} c(\delta)}$.

Desta forma, temos ||$x_{0}||<\rho_{1} \rightarrow x\left(t, t_{0}, x_{0}\right) \rightarrow 0$ com $t_{0} \geq T_{0}$. De fato: afirmamos de início que existe $t_{1} \epsilon$ $\left[t_{0}, t_{0}+T\right]$ tal que ||$x\left(t_{1}, t_{0}, x_{0}\right)||<\delta$.

Caso conträrio teríamos ||$x\left(t, t_{0}, x_{0}|| \geq \delta\right.$ para cada $t \in\left[t_{0}, t_{0}+T\right]$.

\section{Então:}

$$
\begin{aligned}
& v(t, x)-v\left(t_{0}, x_{0}\right)=\int_{t_{0}}^{t} v(s, x(s)) d s \leq-\int_{t_{0}}^{t} c(|x|) d s+ \\
& +\int_{t_{0}}^{t} h(s) d s \leq-c(\delta(\varepsilon))\left(t-t_{0}\right)+\int_{t_{0}}^{t} h(s) d s \rightarrow v(t, x) \leq \\
\leq & v_{0}-c(\delta)\left(t-t_{0}\right)+\int_{t_{0}}^{t} h(s) d s .
\end{aligned}
$$




$$
\begin{aligned}
& \text { Pondo } t=t_{0}+T \text { vem: } \\
& 0 \leq v(t, x) \leq v_{0}-c(\delta) \cdot T+\int_{t_{0}^{t}+T}^{t_{0}} h(s) d s \leq g\left(\rho_{1}\right)- \\
& -\left(g\left(\rho_{1}\right)+\frac{\varepsilon}{2}\right)+\int_{t_{0}}^{t_{0}+T} h(s) d s+\int_{t_{0}}^{t_{0}+T} h(s) d s \geq \frac{\varepsilon}{2},
\end{aligned}
$$

- que contradiz novamente o fato de $h \in M_{1}$.

Desta forma, existe $t_{7} \in\left[t_{0}, t_{0}+T\right]$ tal que

$$
|| x\left(t_{1}, t_{0}, x_{0}\right) \|<\delta \stackrel{2.4 .3}{\rightarrow}|| x\left(t, t_{0}, x_{0}\right) \mid<\varepsilon
$$

para $t \geq t_{1}$, a forciori para $t \geq t_{0}+T \rightarrow x=0$ satisfaz D.2. (2.4.4)

$(2.4 .1)$ e (2.4.4) asseguram que $x=0$ satisfaz a defi nição D. 3 .

Como consequência de um teorema recíproco de existên cia de funcionais de Liapunov e do teorema (2.4) decorre o:

TEOREMA 2.5:

Assumimos que o sistema $\dot{x}=f(t, x)$ (I) tem a origem como um ponto uniformemente assintoticamente estável; com $f(t, x)$ localmente Lipschtz em $x$, uniformemente com relação a t. Logo o sistema $\dot{x}=f(t, x)+g(t, x)$ (II) è Eventualmente as sintoticamente uniformemente estável se $|g(t, x)| \leq h(t)$ para ||$x||<r_{0}, \quad r_{0}>0$ e $h(t) \in M_{0}$.

D. Como foi referido nas preliminares deste capítulo usa remos nesta verificação um lema que está transcrito no apêndi ce destas notas.

De acordo com o lema [ap.], o sistema II admite um fun cional $v$ satisfazendo $\dot{v}_{I I}(t, x) \leq-c(|x|) v(t, x)+K \mid g(t, x)$ p pa ra $t \geq 0$ e ||$x|| \leq r_{1}$.

Agora, dado que $x=0$ (origem do sistema I) satisfaz 
L.2, o item (b) do Teorema [ap.] assegura:

$-c(|x|) v(t, x) \leq-c(|x|)|x|$ para $t \in J$ e ||$x||<r_{1}$

Desta forma:

$$
\dot{v}_{I I}(t, x) \leq-c(|x|)|x|+K|g(t, x)| \text {. }
$$

Agora, para ||$x||<r_{0}$ por hipōtese é vālida a majora ção $|g(t, x)| \leq h(t) \operatorname{com} h \in M_{0^{\circ}}$.

Escolhendo $r=\min \left\{r_{0}, r_{1}\right\}$ temos que:

$$
|| x||<r \underset{2.5 .3}{\stackrel{2.5 .1}{\rightarrow}} \dot{v}(t, x) \leq-c(|x|)|x|+K h(t)
$$

$\operatorname{com} h \in M_{0}$

Observamos com is to que:

$$
1-h \in M_{0} \rightarrow h_{1}=K h \in M_{0}
$$$$
\rightarrow \dot{v}_{I I}(t, x) \leq
$$

$|-u(x)=c(|x|)| x \mid$ é definida positiva

$\leq u(x)+h_{1}(t)$ e portanto a condição (c) do Teorema (2.4) es tá satisfeita.

As demais exigências (a) e (b) do Teorema (2.4) estão contidas no item (a) do teorema do apêndice.

Logo, estamos nas condições do Teorema (2.4) $\rightarrow x=0$ do sistema II satisfaz D.3 e a prova se completa.

Este teorema estabelece que se o sistema perturbado se aproxima de forma suficientemente rápida do sistema não per turbado: o qual "a priori" sabe-se que é uniformemente assinto ticamente estável, então ele é eventualmente uniformemente as sintoticamente estável. 


\section{CAPITULO 3}

RELAÇŌES COM A TEORIA

DE LIAPUNOFF

A idéia central deste capítulo é motivada da seguinte pergunta: "sob quais condições" o requisito "ser eventualmente estāvel" recai num caso clāssico de estabilidade segundo Liapu noff.

Tal problema é abordado com os teoremas (3.1.1), $(3.1 .2),(3.2 .1)$ e $(3.2 .2)$ deste capítulo.

Como deveremos estabelecer comparações com a teoria de Liapunoff se faz mister transcrever abaixo ao menos aquelas definições necessārias ao nosso propōsito.

E suposto em tais definições que $x=0$ é um ponto de equilíbrio do sistema $I$, isto é, $f(t, 0)=0$ para cada $t \in J=$ $=[0, \infty)$.

L.1 - A solução $x=0$ de I é dita uniformemente estāuel, se pạ ra cada $\varepsilon>0$ e $t_{0} \in J$, existe um $\delta=\delta(\varepsilon)>0$ tal que se ||$x_{0}||<\delta$ então ||$x(t)||<\varepsilon \quad \forall t \geq t_{0}$.

L.2 - A solução $x=0$ de I é dita uniformemente assintoticamen te estāuel se satisfaz $L .1$, e existe $\delta_{0}>0$ tal que, pa ra cada $\varepsilon>0, t_{0} \in J$ e ||$x_{0}||<\delta_{0}$ existe $T=T(\varepsilon) \geq 0$ tal que se $t \geq t_{0}+T$; então ||$x\left(t, t_{0}, x_{0}\right)||<\varepsilon$. 


\section{1 - ESTABILIDADE EVENTUAL UNIFORME}

TEOREMA $3.1 .1:$

Se a origem é ponto de equilíbrio do sistema I então estabilidade eventual uniforme da origem é equivalente a esta bilidade uniforme.

Demonstração:

Queremos verificar que se $f(t, 0)=0$; então D.1 é equi valente a L.I.

$$
\begin{aligned}
& \text { a) } L .1 \rightarrow D .1 \\
& x=0 \text { satisfaz L. } 1 \rightarrow \text { dado } \varepsilon>0, \exists \delta=\delta(\varepsilon)>0 \\
& || x\left(t_{0}\right)\left\|<\delta \rightarrow|| x\left(t, t_{0}, x_{0}\right)\right\|<\varepsilon \quad \forall t \geq t_{0} \\
& \text { Tomemos } T=T(\varepsilon)=T_{0} \geq 0 \text { qualquer fixado. Logo, } \\
& \text { teremos: dado } \varepsilon>0 \exists \delta=\delta(\varepsilon)>0, T=T(\varepsilon) \text { tal que se } \\
& || x_{0}\left\|<\delta \rightarrow|| x\left(t, t_{0}, x_{0}\right)\right\|<\varepsilon \quad \forall t \geq t_{0} \geq T . \\
& \text { Desta forma } x=0 \text { satisfaz D.l. } \\
& \text { b) D.1 } \rightarrow L .1 \\
& x=0 \text { satisfaz D.I } \rightarrow \text { dado } \varepsilon>0, \exists \delta=\delta(\varepsilon)>0, \\
& \delta<\varepsilon \text { e } T=T(\varepsilon)>0||\left|x_{0}\right| \mid<\delta \text { então }|| x\left(t, t_{0}, x_{0}\right) \mid<\varepsilon \\
& \forall t \geq t_{0} \geq T \cdot(1)
\end{aligned}
$$

Agora, como estamos supondo válida para o sistema I as condições de dependência contínua de cada solução, em re lação aos dados iniciais, então:

Dado $\delta>0$, existe $\delta_{1}=\delta_{1}(\delta)>0$ tal que ||$x_{0}||<$ $<\delta_{1} \rightarrow|| x(t)||<\delta \quad \forall t$ satisfazendo $0 \leq t_{0} \leq t \leq T$. Como $\delta=\delta(\varepsilon)$ então $\delta_{1}=\delta_{1}(\varepsilon)$.

Logo, dado $\varepsilon>0, \exists \delta_{1}=\delta_{1}(\varepsilon)>0$ tal que se 


$$
\begin{gathered}
|| x_{0} \|<\delta_{1} \rightarrow|| x\left(t, t_{0}, x_{0}\right)|<\varepsilon \ldots t| 0 \leq t_{0} \leq t \leq T . \\
\text { Desta forma dois casos podem ocorrer: }
\end{gathered}
$$$$
\mid-t \geq t_{0} \geq T \text { então }|| x_{0}||<\delta \text { vale(1) }+|| x\left(t, t_{0}, x_{0}\right)||<\varepsilon
$$$$
1-t \geq t_{0} \geq 0 \text { com } t_{0} \leq T \text { se }|| x\left(t_{0}\right)||<\delta_{1}<\delta
$$

e em particular ||$x(T)||<\delta_{1}$, então vale(2) $\rightarrow|| x\left(t, t_{0}, x_{0}||<\right.$ $<\delta<\varepsilon$.

$$
\text { Então para } t \geq t_{0} \geq 0,|| x_{0}\left\|<\delta_{1} \rightarrow\right\| x\left(t, t_{0}, x_{0} \|\right.
$$

$<\varepsilon$.

Assim sendo $x=0$ satisfaz L.I e a prova está com pleta.

E bom ressaltar que se a origem não é ponto de equilíbrio do sistema; então D.l $\nrightarrow$ L.l como mostra o seguin te exemplo.

E.4 - Consideremos o. sistema $\dot{x}=\phi(t) \operatorname{com} \phi(t)>0 t \in J$ e $\int_{0}^{\infty} \phi(s) d s<\infty$. A solução deste sistema satisfaz D.l mas não satisfaz L.I.

De fato: a solução do sistema é dada por:

$x(t)=-\int_{t}^{\infty} \phi(s) d s+c(1)$.

Impondo a condição $x\left(t_{0}, t_{0}, x_{0}\right)=x_{0}$ vem $\rightarrow \quad x_{0}=-$ $-\int_{t_{0}}^{\infty} \phi(s) d s+c \rightarrow c=x_{0}+\int_{t_{0}}^{\infty} \phi(s) d s(2)$.

(2) em (1) $\rightarrow x\left(t, t_{0}, x_{0}\right)=x_{0}+\int_{t_{0}}^{t} \phi(s) d s t \geq t_{0}$

Sendo que $\phi$ possue integral convergente em $J \rightarrow$ dado $\varepsilon>0$ existe $T=T(\varepsilon)>0 \mid \int_{t}^{\infty} \phi(s) d s<\frac{\varepsilon}{2}$ para $t \geq T .(4)$

Portanto; dado $\varepsilon>0, \exists \delta=\delta(\varepsilon)=\frac{\varepsilon}{2}$ tal que se 


$$
\begin{aligned}
& || x_{0}||<\delta \underset{(3)}{\rightarrow}\left|x\left(t, t_{0}, x_{0}\right) \leq\right| x_{0}|+| \int_{t_{0}}^{t} \phi(s) d s \mid<\delta+ \\
& \quad+\int_{t_{0}}^{t} \phi(s) d s=\frac{\varepsilon}{2}+\int_{t_{0}}^{t} \phi(s) d s<\frac{\varepsilon}{2}+\int_{T}^{\infty} \phi(s) d s<\text { (4) } \\
& \quad<\frac{\varepsilon}{2}+\frac{\varepsilon}{2}=\varepsilon \text { se } t \geq t_{0} \geq T .
\end{aligned}
$$

Desta forma a solução dada pela expressão (3) verifí ca a definição D.l.

A não validade da definição L.l está ligada ao sim ples fato que sendo $\phi(t)>0$ para $t \in J$, a origem $x=0$ não se rá um ponto de equilíbrio do sistema dado.

TEOREMA 3.1.2:

Se o sistema I for autônomo, então estabilidade even tual uniforme da origem é equivalente a estabilidade uniforme.

Demonstração:

Vamos verificar que se $f(t, x)=f(x)$ e a origem è eventualmente uniformemente estāvel então recessariamente $f(0)=0$.

De fato se $f(0) \neq 0 \rightarrow\left\|\left(f_{1}(0), f_{2}(0), \ldots, f_{n}(0)\right)\right\| \geq$ $\geq a>0 \rightarrow \exists i \in\{1,2, \ldots, n\}$ e $\beta>0|| f_{i}(0) \mid>\beta>0$.

A continuidade da $f$ garante a existência de um real $r>0 \quad \forall x \in B_{r}(0) C I^{n}$ temos $\left|f_{i}(x)\right|>\frac{\beta}{2}>0$.

Sendo que a origem $x=0$ satisfaz D.I $\rightarrow$ Dado $r>0$, $\exists \delta=\delta(r)>0$ e $T=T(r)>0$ tal que ||$x_{0}||<\delta \rightarrow|| x\left(t, t_{0}\right.$, $x_{0} \|<r$ para $t \geq t_{0} \geq T$.

Se colocarmos a solução de I na forma vetorial, e con siderarmos a componente de índice $i$ teremos:

$$
x_{i}(t)=x_{i}^{0}+\int_{t_{0}}^{t} f_{i}\left(x_{1}(s), \ldots, x_{n}(s)\right) d s .
$$


A relação (1) nos leva a considerar dois casos:

$$
\begin{aligned}
& 1-f_{i}\left(x_{1} \ldots x_{n}\right)>\beta \mid 2 \\
& \left|-f_{i}\left(x_{1} \ldots x_{n}\right)<-\beta\right| 2
\end{aligned}
$$

Ocorrendo o primeiro deles a igualdade (3) fornece: $x_{i}(t)>x_{i}^{0}+\frac{\beta}{2}\left(t-t_{0}\right) \rightarrow x_{i}(t) \rightarrow \infty$ quando $t \rightarrow \infty$. De forma análoga se ocorre o segundo caso, então: $x_{i}(t)<x_{i}^{0}-\frac{\beta}{2}\left(t-t_{0}\right) \rightarrow x_{i}(t) \rightarrow-\infty$ quando $t \rightarrow \infty$. Em ambos os casos fica contrariada a definição D.l dạ da pela relação (2) .

Logo; $f(0)=0 \rightarrow x=0$ é um ponto de equilíbrio do sistema I $\frac{\text { Teor. }}{3.1 .1} D .1 \leftrightarrow L .1$ e a prova está completa.

\section{2 - ESTABILIDADE EVENTUAL ASSINTOTICO - UNIFORME}

TEOREMA 3.2.1:

Se a origem é ponto de equilíbrio do sistema I, então estabilidade eventual assintōtica uniforme é equivalente a es tabilidade assintótica uniforme.

Demonstração:

Queremos verificar a equivalência D.3 $\rightarrow$ L.2.

a) $L .2 \rightarrow D .3$

Se vale a definição L.2 $\rightarrow x=0$ satisfaz L.I $\frac{\text { Teor. }}{3.1 .1}$ $x=0$ satisfaz D.I.(1)

$$
\text { Agora } x=0 \text { é assintoticamente estável } \rightarrow \text { Dado }
$$

$\varepsilon>0, \exists \delta_{0}>0, T=T(\varepsilon)>0$ tal que:

$$
|| x_{0}||<\delta_{0} \rightarrow|| x\left(t, t_{0}, x_{0}\right)||<\varepsilon \forall t \geq t_{0}+T .
$$


A fim de assegurar a existência do $T_{0}$ da definição D.2 tomamos $T_{0}=0 \rightarrow x=0$ satisfaz D.2. (2)

(1) e (2) $\rightarrow x=0$ satisfaz D.3.

b) $D .3 \rightarrow L .2$

A primeira parte desta verificação è inteiramente análoga àquela que realizamos na implicação anterior.

Sendo que $x=0$ verifica D. $\rightarrow x=0$ verifica D.2 $\rightarrow$ dado $\varepsilon>0$, existem nümeros positivos $\delta_{0}, T_{0}, T=T(\varepsilon)$ tais que: se ||$x_{0}|| \leq \delta_{0} \rightarrow|| x\left(t, t_{0}, x_{0}\right)||<\varepsilon \quad t \geq t_{0}+T$ $\operatorname{com} t_{0} \geq T_{0}$

Logo; dado $\varepsilon>0, \quad \exists \delta_{0}$ e $T=T(\varepsilon)$ tais que: se ||$x_{0}||<\delta_{0} \rightarrow|| x\left(t, t_{0}, x_{0}\right)||<$ para $t \geq t_{0}+T \rightarrow x=0$ sa tisfaz a definição L.2.

TEOREMA 3.2.2:

Se o sistema I for autônomo então estabilidade even tual assintōtica uniforme da origem é equivalente a estabilida de assintōtica uniforme.

D. - A dificuldade essencial concentrada aqui seria a de verificar que se $f(t, x)=f(x)$, e $x=0$ satisfaz D.3 então $f(0)=0$.

Este tipo de dificuldade jā foi contornado no Teore ma 3.1.2. Os demais detalhes decorrem de um uso natural de im plicações lançando-se mão dos teoremas $3.1 .2,3.2 .1$ e 3.2.2. 


\section{CAPITULO 4}

o que tencionamos com este parágrafo é: dentro de dẹ terminadas condições às quais o sistema é submetido, obter re giões nas quais poderemos assegurar estabilidade do tipo even tual.

Os dois primeiros resultados seguem a linha proposta pelo teorema 2.5 do capítulo 2. A aplicação a ser proposta es tā fundamentada essencialmente no ūltimo teorema deste capĩu 10 .

TEOREMA 4.1:

Seja $M$ uma parte compacta do $\mathbb{R}^{n}$ contendo a origem. Se ja $N$ C $M$ um subconjunto com a propriedade: As soluções da equa ção que partem de $N$ para um tempo $t_{0} \geq T$ permanecem logo após em $M$.

Em adição vamos supor a existência de fúnções $V(t, x)$, $v(x)$ e $w(x)$, a valores reais satisfazendo:

$\|(a) V(t, x) \rightarrow v(x)$ uniformemente em $x$ para $t \rightarrow \infty$; $x \in M$.

$\|(b \mid \dot{V}(t, x) \rightarrow-w(x)$ uniformemente em $x$ para $t \rightarrow \infty$; $x \in M$.

$\|$ (c) $v$ e $w$ são definidas positivas.

Então; existe $T_{0}>0$ tal que $x\left(t, t_{0}, x_{0}\right) \rightarrow 0$ quando $t \rightarrow \infty$ para cada $x_{0} \in N, t_{0} \geq T_{0}$.

D. Dado $\varepsilon>0$, seja $m=$ inf $v(x)$; logo $m>0$. $\| x||=\varepsilon$. 
1 - De $(a)$ dado $m=m(\varepsilon)>0, \exists T_{1}=T_{1}(\varepsilon)$ tal que $t \geq T_{1} \rightarrow|V(t, x)-v(x)|<\frac{m}{2} \operatorname{com}|| x||=\varepsilon$. Assim $V(t, x)>$ $>\frac{m}{2}$ na superficie da esfera $S_{\varepsilon}(0), t \geq T_{1}$.

- De $(c)$ dado que $v(0)=0(v e K)$ e $v$ é contínua $\rightarrow \exists \delta=\delta(\varepsilon)>0$ e $T_{2}=T_{2}(\varepsilon) \mid t \geq T_{2}$ e ||$x|| \leq \delta \rightarrow V(t$, $x)<\frac{m}{2}$.

De fato: $v(0)=0$ e $v$ contínua $\rightarrow$ dado $m(\varepsilon)>0$, existe $\delta=\delta(\varepsilon) \quad \mid v(x) \leq \frac{m(\varepsilon)}{4}$ se ||$x|| \leq \delta$.

Agora $V \rightarrow v$ então $\lim _{t \rightarrow \infty} V(t, 0)=v(0)=0 \rightarrow$ dado $m(\varepsilon)>0, \exists T_{2}=T_{2}(\varepsilon)>0$ tal que $t \geq T_{2} \rightarrow|V(t . x)-v(x)|<$ $<\frac{m(\varepsilon)}{4} \rightarrow \nabla(t, x)<v(x)+\frac{m(\varepsilon)}{4}<\underset{4.3}{<1.3} \frac{m(\varepsilon)}{4}+\frac{m(\varepsilon)}{4}=\frac{m(\varepsilon)}{2} \mathrm{pa}$ ra $t \geq T_{2}$ e ||$x|| \leq \delta$.

1- De (c) dado que $w \in K$ considero

$$
\beta=\delta \leq|| x \mid \leq \varepsilon w(x)=\beta(\varepsilon)>0 \text {. }
$$

Desta forma tendo em consideração o item $(b) \rightarrow \exists T_{3}=T_{3}(\varepsilon)$ tal que $\dot{V}(t, x)<-\frac{\beta}{2}$.

$$
\text { De fato: } \dot{V} \rightarrow-w \rightarrow \text { dado } \frac{\beta}{2}>0 \quad \exists \quad T_{3}=T_{3}(\varepsilon)>
$$

$>0|| \dot{V}(t, x)+w(x) \mid<\frac{\beta}{2}$ se $t \geq T_{3}$.

$$
\text { Logo } \dot{V}(t, x)<-w(x)+\frac{\beta}{2} \leq-\beta+\frac{\beta}{2}=-\frac{\beta}{2}
$$

para $t \geq T_{3}$ e $\delta \leq|| x|| \leq \varepsilon$.

\section{Escolhemos}

$$
\bar{T}=\max _{i \in\{1,2,3\}} T_{i}=\bar{T}(\varepsilon)
$$

e vamos supor que para a solução $x\left(t, t_{0}, x_{0}\right)$ de I tenhamos para algum $t_{1} \geq \bar{T},|| x\left(t_{1}, t_{0}, x_{0}\right) \|<\delta$ e que existam $t_{2}, t_{3}$ satisfa 
zendo $\bar{T} \leq t_{1}<t_{2}<t_{3} \operatorname{com}|| x_{2}\left\|=|| x\left(t_{2}, t_{0}, x_{0}\right)\right\|=\delta_{j}\left\|x_{3}\right\|=$ $=|| x\left(t_{3}, t_{0}, x_{0}\right)\|=\varepsilon e \delta \leq|| x(t)\| \leq \varepsilon \operatorname{para} t \in\left[t_{2}, t_{3}\right]$.

Desta forma de $(4.1 .1)$ e $(4.1 .2)$ vem $V\left(t_{2}, x_{2}\right)<\frac{m}{2}<$ $\left\langle V\left(t_{3}, x_{3}\right) \rightarrow V\left(t_{3}, x_{3}\right)-V\left(t_{2}, x_{2}\right)>0 \rightarrow \exists s \in\left[t_{2}, t_{3}\right]\right.$ tal que $\dot{V}(s, x(s))>0$.

Mas na região anular $S_{\delta}^{\varepsilon}=\left\{x \in I^{n}|\delta \leq||x| \mid \leq \varepsilon\right\} \circ$ item (4.1.4) garante que $\dot{V}(t, x(t))<0 \quad \forall t \geq \bar{T} \geq T_{3}$.

Os itens $(4.1 .5)$ e $(4.1 .6)$ são claramente contraditó rios. Assim, se existe $t_{1} \geq \bar{T}$ tal que ||$x_{1}||=|| x\left(t_{1}, t_{0}, x_{0}\right) \mid<$ $<\delta \rightarrow|| x\left(t, t_{0}, x_{0}\right)||<\varepsilon$ para todo $t \geq t_{1}$. Por conseguinte a solução $x\left(t, t_{0}, x_{0}\right)$ de I satisfaz D.I pelo Teorema 2.1 .

Resta provar o carāter assintótico, ou seja, existe $T_{0}>0$ tal que $x\left(t, t_{0}, x_{0}\right) \rightarrow 0$ quando $t \rightarrow \infty$ para cada $x_{0} \in N$, $t_{0} \geq T_{0}$

Por hipótese; existe $T$ tal que as soluções que partem de $N$ para um tempo $t_{0} \geq T$ permanecem logo após em $M$.

Afirmamos que para todo $T_{0} \geq T$ é satisfeita a exigên cia dada por (4.1.7).

De fato: vamos supor $t_{0} \geq T_{0}$ e que $\nexists t \geq t_{0} \geq T_{0}$ tal que $x\left(t, t_{0}, x_{0}\right) \in S_{\delta}(0)$.

Desta forma, $\forall t \geq t_{0}$ temos $x\left(t, t_{0}, x_{0}\right) \notin s_{\delta}(0) \rightarrow$ $\rightarrow|| x\left(t, t_{0}, x_{0}\right) \mid \geq \delta$.

Um raciocínio inteiramente análogo àquele efetuado na primeira parte desta demonstração nos conduz a um absurdo.

Logo, $\exists t \geq t_{0} \geq T_{D}$ tal que $x\left(t, t_{0}, x_{0}\right) \in S_{\delta}(0)$ $\rightarrow|| x\left(t, t_{0}, x_{0}\right)||<\delta \stackrel{D_{.1}}{\rightarrow}|| x\left(t, t_{0}, x_{0}\right)||<\varepsilon, t \geq t_{0} \geq T_{0} \rightarrow$ $\rightarrow|| x\left(t, t_{0}, x_{0}\right)||<\varepsilon$ para $t \geq t_{0}+\bar{T}$ e portanto a prova se completa. 
TEOREMA 4.2:

Assumimos que $M$ limitado no $I R^{n}$ é dado por $v(x) \leq L$ tal que são vālidas as conđições (a), (b) e (c) do Teorema 4.1 Vamos supor que para cada $\delta>0 M_{\delta}$ è dado por $v(x) \leq L-\delta$. Logo para cada $\delta>0$, existe $T_{\delta}$ tal que $x_{0} \in M_{\delta}$ e $t_{0} \geq T_{\delta}$ en tão $x\left(t, t_{0}, x_{0}\right) \rightarrow 0$ quando $t \rightarrow \infty$.

D. - De fato; dado que $v(0)=0, v$ é contínua monótona cres cente, então $M=\left\{x \in \mathbb{R}^{n} \mid v(x) \leq L\right\}=v^{-1}([0, L])$.

Portanto $M$ é fechado, e sendo que por hipōtese $M$ è $1 \underline{i}$ mitado $\rightarrow M \bar{e}$ compacto.

Ainda, para cada $\delta>0 \rightarrow M_{\delta}=\left\{x \in I R^{n} \mid v(x) \leq L-\right.$ $-\delta\}=v^{-1}([0, L-\delta]) \mathrm{C} v^{-1}([0, L])=M \rightarrow M_{\delta} \mathrm{C} M$.

As condições $(4.2 .1)$ e $(4.2 .2)$ e mais a hipōtese asse guram que para cada $\delta>0$ o conjunto $M_{\delta}$ se encontra nas condi ções do Teorema $4.1 \rightarrow \exists T_{\delta}$ tal que $x_{0} \in M_{\delta}$ e $t_{0} \geq T_{\delta}$ então $x\left(t, t_{0}, x_{0}\right) \rightarrow 0$ quando $t \rightarrow \infty$.

TEOREMA 4.3:

Para o sistema:

II

$$
\| \dot{x}=f(t, x, y) \quad x \in \mathbb{R}^{p}
$$

$\| \dot{y}=g(t, x, y) \quad y \in \mathbb{I R}^{q}$,

existe uma função escalar $v(x, y)$ com as seguintes pro priedades :

a) $v(x, y)$ é definida positiva e tem derivadas parciais continuas para todo $x$ e $y$.

b) $v(x, y) \rightarrow \infty$ quando $\left.|| x\right|^{2}+\left.|| y\right|^{2}+\infty$. 
c) $\dot{v}(x, y) \leq-w(x)+h(t) q(x, y)$ onde $w(x)$ è definida positiva; $q(x, y)$ è continua no par $(x, y)$ e $h \in M_{0}$.

d) $f(t, x, y)$ è limitada em $J \times K$, onde $K$ : compacto do $\mathbb{I R}^{n}, n=p+q$.

Então a origem $x=0, y=0$ do sistema satisfaz D.1 e correspondente a cada $r>0$, existe $T_{0}$ tal que se $\left\|x_{0}\right\|^{2}+$ $+\left.|| y_{0}\right|^{2}<r^{2}$ para $t_{0} \geq T_{0}$ implica que $y(t)$ é limitada para $t \geq t_{0}$ e $x(t) \rightarrow 0$ quando $t \rightarrow \infty$.

Observação:

$E$ bem conhecido que se $B_{\delta_{i}}(0) C \mathbb{R}^{p}$ é um sistema fun damental de vizinhanças do zero no $\mathbb{I R}^{p}$, e $B_{\varepsilon_{i}}$ (0) $\mathrm{C} \mathbb{I R}^{q}$ è 0 anā logo correspondente no $\mathbb{I R}^{q}$ então $B_{\delta_{i}}(0) \times B_{\varepsilon_{i}}{ }^{(0)} \mathrm{C} \mathbb{I R}^{n}$ onde $n=$ $=p+q, \overline{\mathrm{e}}$ um sistema fundamental de vizinhanças do zero no $\mathbb{R}^{n}$. Assim no transcorrer desta verificação, se $z(t)=$ $=(x(t), y(t))$ è qualquer solução de II dizer que $z(t) \epsilon$ $\in B_{R}(0) C I R^{n}$ è equivalente a dizer que $\|z(t)\|<R$, onde || || é qualquer Norma conveniente no $I^{n}$.

Dado que ||$x|| \leq|| z|| \rightarrow x(t) \in B_{R}(0)$ onde $B_{R}(0)$ c $\mathbb{R}^{p}$. A demonstração do teorema será feita por etapas.

\section{$1.10,0)$ satisfaz 0.1 .}

Se $z=(x, y)$ é um vetor no $I^{n}$ então $\dot{z}=(\dot{x}, \dot{y})$ onde $\dot{x}$ e $\dot{y}$ são regidos pelas equações do sistema II.

o nosso trabalho agora será concentrado em colocar a origem $z=0$ de II nas condições do Teorema 2.2.

Observamos que da condição (c) temos:

$$
\begin{aligned}
\dot{v} \leq- & w(x)+h(t) q(x, y)=-w(x)+h(t) q(z) \leq h(t) q(z) \leq \\
& \leq|h(t)||q(z)| .
\end{aligned}
$$


Agora para os fins que nos propomos, $q(z)$ pode ser sü posta limitada; ou seja $|q(z)| \leq L$ para algum $L>0$, pois $q$ è continua num compacto conveniente.

De (4.3.1) concluímos então que:

$\dot{v} \leq|h(t)||q(z)| \leq|h(t)| L=\lambda(t)$.

E fácil constatar que se $h \in M_{0} \rightarrow \lambda=|h| L \in M_{0}$ com I constante.

Ainda; dado que por $(a) v(z)$ é definida positiva, en tão $v(0)=0$ e $v$ é positiva fora da origem. Logo:

$1-v(0)=0 \rightarrow v(z) \leq a(|| z||) \operatorname{com} a \in K$, isto è, $v$ admite extremo superior infinitésimo. (4.3.3) [ap. 3] .

L $v$ definida positiva $\rightarrow v(z) \geq b(|| z||)$ com $b \in$ $\boldsymbol{E} K$.

As condições $(4.3 .2),(4.3 .3)$ e $(4.3 .4)$ colocam o fun cional $v(z)$ nas condições do T.2, onde o papel de $\phi(t)$ no teo rema è aqui representado por $\lambda(t)$.

Decorre então que $z=0$ satisfaz a definição D.l.

2.) Limitação das soluções de II

Afirmamos que $\forall x>0, \exists T_{0}^{(1)}$ tal que para toda solu ção $z(t)=(x(t), y(t))$ de II partindo de $z_{0}=z\left(t_{0}\right) \in B_{r}(0)$, $t_{0} \geq T_{0}^{(1)}$ é limitada para $t \geq t_{0}$.

De fato: negando a afirmação acima obtemos: $\forall T_{O}$, $\exists r>0, \exists$ solução $z(t)$ de II tal que $z_{0} \in B_{r}(0)$ mas $z(t)$ não é limitada para $t \geq t_{0} \geq T_{0}$.

Tomemos

$$
L_{1}={ }_{|| x||^{2}}^{\max }+|| y||^{2}=r^{2}
$$


Dado que $v(x, y) \rightarrow \infty$ quando $\left.|| x\right|^{2}+|| y||^{2} \rightarrow \infty$, então $\exists R>0$ suficientemente grande tal que

$$
L_{2}=L_{2}(R)=\min _{\left\|\left.x\right|^{2}+|| y\right\|^{2}=R^{2}}^{v(x, y)}
$$

satisfaz $\beta(R)=L_{2}-L_{1}>0$.

Em adição vamos supor $t_{1}$ e $t_{2}$ tempos satisfazendo $T_{0} \leq t_{0}<t_{1}<t_{2} \operatorname{com}|| x\left(t_{1}\right)\left\|^{2}+|| y\left(t_{1}\right)\right\|^{2}=r_{2}, \|\left.\left. x\left(t_{2}\right)\right|_{1}\right|^{2}+$ $\left\|y\left(t_{2}\right)\right\|^{2}=R^{2}$ e $z(t) \in S_{r}^{R}$ para $t \in\left[t_{1}, t_{2}\right]$.

Daqui para frente a mesma linha de idéias realizada na 1 a parte da verificação do teorema 4.1 nos conduz a um ab surdo.

Logo $\forall r>0, \exists T_{0}^{(1)}$ tal que para toda solução $z(t)$ de II satisfazendo $z_{0} \in B_{p}(0), t_{0} \geq T_{0}^{(1)}, z(t)$ é limitada para $t \geq t_{0}$

Em particular, dado que ||$y(t) \| \leq|| z(t)||$ então pạ ra cada $r>0$ existe $T_{0}^{(1)}$ tal que $z_{0} \in B_{r}(0)$ então $y(t)$ é limi tada para $t \geq t_{0}$.

E evidente que $\circ T_{0}^{(1)}$ assim obtido é função de cada $r>0$ dado, isto é, $T_{0}^{(1)}=T_{0}^{(1)}(r)$.

Como cada solução partindo da bola de raio $r>0$ do sistema II é limitada, resta somente verificar que $x(t) \rightarrow 0$ quan do $t \rightarrow \infty$, ou seja: $\forall r>0, \exists T_{0} \mid t_{0} \geq T_{0}$ e $\left\|z_{0}\right\|^{2}<r^{2} \rightarrow$ $\rightarrow \exists R>0|| \mid z(t) \|^{2} \leq R^{2}$ e $x(t) \rightarrow 0$ quando $t \rightarrow \infty$.

Inicialmente observamos que:

$$
\begin{aligned}
& \text { (a) } h \in M_{0} \rightarrow e^{-t} \int_{0}^{t} e^{s}|h(s)| \text { as } \rightarrow 0 \\
& \text { quando } t \rightarrow \infty \text {. }
\end{aligned}
$$


(b) ||$z(t) \|^{2} \leq R^{2}+\exists L>0$ tal que $|q(x, y)| \leq L$ pois $q$ é contínua no par $(x, y)$.

Definimos $U$ uma nova função de Liapunoff dada por: $U=U(t, x, y)=e^{-\int \frac{t}{0} e^{(s-t)}|h(s)| d s}\{v(x, y)+L\}$ onde $v(x, y)$ é o funcional associado ao sistema II.

$U(t, x, y)$ satisfaz as seguintes propriedades:

$$
\begin{aligned}
& 1-\quad v(t, x, y) \geq 0 \\
& 1-\dot{U}(t) \leq-\frac{1}{e} w(x)
\end{aligned}
$$

A primeira delas é óbvia. Verifiquemos então a segun da.

\section{Temos :}

$$
\begin{aligned}
\dot{U}(t)= & -e^{-\int_{0}^{t} e^{(s-t)}|h| d s}|h(t)|\{v+L\}+\dot{v} e^{-\int_{0}^{t} e^{(s-t)}|h| d s} \\
= & e^{-\int_{0}^{t} e^{(s-t)}|h| d s}\{-|h(t)|(v+L)+\dot{v}\} \leq \\
& e^{-\int_{0}^{t} e^{(s-t)}|h| d s}\{-|h| v-|h| L-w(x)+h q(x, y)\} \\
\leq & -e^{-\int_{0}^{t} e^{(s-t)}|h| d s} \cdot w(x) .
\end{aligned}
$$

Em (*) foi usado que $|h| v \geq 0$ e ainda que $h q$ - $|h| L \leq 0$; observando que $h=h(t)$ e $v=v(x, y)$.

Agora, de (4.3.5) vem: Dado $\varepsilon=1$ existe $T_{0}^{i 2)}=T_{0}^{(2)}$ (1) tal que se $t \geq T_{0}^{(2)}$ então $\int_{0}^{t} e^{(s-t)}|h| d s<1$

$$
\begin{aligned}
& \text { Ponhamos } f(t)=\int_{0}^{t} e^{(s-t)}|h(s)| d s . \\
& \text { Logo, de (4.3.10) } t \geq T_{0}^{(2)} \rightarrow f(t)<1 \rightarrow e^{f(t)}<e \rightarrow
\end{aligned}
$$

$\rightarrow e^{-f(t)}>e^{-1} \rightarrow-e^{-f(t)}<-e^{-1}=-\frac{1}{e}$. 
Esta ültima desigualdade combinada com (4.3.9) nos le va a $\dot{U}(t) \leq-\frac{1}{e} w(x)$ para $t \geq T_{0}^{(2)}$.

Escolhemos $T_{0}=\max \left\{T_{0}^{(1)}, T_{0}^{(2)}\right\}$ e afirmamos que: se $z_{0} \in B_{r}(0) \quad t_{0} \geq T_{0}$ então:

$$
\begin{aligned}
& 1-y(t) \text { è limitada para } t \geq t_{0} \\
& 1-x(t) \rightarrow 0 \text { quando } t \rightarrow \infty .
\end{aligned}
$$

A primeira destas afirmações é imediata pois é vãlida para $t \geq t_{0} \geq T_{0}^{(1)}$; portanto fica verificada para $T_{0} \geq T_{0}^{(1)}$. Passemos à segunda.

De fato se $x(t) \neq 0$ quando $t \rightarrow \infty \rightarrow \exists \varepsilon_{0}$ e sequência $\left\{t_{n}\right\}, t_{n} \rightarrow \infty$ quando $n \rightarrow \infty$ tal que ||$x\left(t_{n}\right) \mid \geq \varepsilon_{0}$.

Logo:

$$
\begin{aligned}
& || x(t)-x\left(t_{n}\right)\|=\| \int_{t_{n}}^{t} \dot{x}(s) d s||=\| \int_{t_{n}}^{t} f(s, x(s), y(s) d s|| \leq \\
& \leq M\left(t-t_{n}\right)
\end{aligned}
$$

Usamos acima a hipótese (d) do Teorema, ou seja, $f(t, x, y)$ è limitada em $J \times K, \quad K$ C $\mathbb{R}^{n} K$ : compacto.

Da relação ||||$x||-|| y|||| \leq|| x-y||$ e da des $\underline{i}$ gualdade (4.3.11) concluímos que

$$
\begin{aligned}
|| x(t)|| & \geq-M\left(t-t_{n}\right)+|| x\left(t_{n}\right)|| \geq-M\left(t-t_{n}\right)+\varepsilon_{0}(4.3 .12) \\
& \text { Tomemos } I_{n}=\left[t_{n}, t_{n}+\frac{\varepsilon_{0}}{2 M}\right] \text { e escolhemos } t_{1} \geq T_{0} \cdot \mathrm{se}
\end{aligned}
$$
necessário passando a uma subsequência podemos supor os $I_{n}$ to dos disjuntos.

Assim

$$
\begin{aligned}
t \in I_{n} \rightarrow & t-t_{n} \leq \frac{\varepsilon_{0}}{2 M} \rightarrow-\left(t-t_{n}\right) \geq-\frac{\varepsilon_{0}}{2 M} \rightarrow-M\left(t-t_{n}\right) \geq \\
& \geq-\frac{\varepsilon_{0}}{2} \\
& \left.\operatorname{se} t \in I_{n} \frac{(4.3 .12)}{(4.3 .13)}|| x(t)|| \geq \frac{\varepsilon_{0}}{2} .3 .13\right)
\end{aligned}
$$


Como $w$ é definida positiva dado $\frac{\varepsilon_{0}}{2}($ fixo), $\exists \sigma>0$ se ||$x(t) \| \geq \frac{\varepsilon_{0}}{2}+w(x) \geq \sigma>0$.

Por conseguinte; a integração de $\dot{U}(t)$ no intervalo $\left[t_{1}, t_{N}\right]$ onde $t_{1}$ e $t_{N}$ são pontos da sequência $\left\{t_{n}\right\}$ fornece:

$$
\begin{aligned}
& U\left(t_{N}+\frac{\varepsilon_{0}}{2 M}, x\left(t_{N}+\frac{\varepsilon_{0}}{2 M} 1, y\left(t_{N}+\frac{\varepsilon_{0}}{2 M}\right)-U\left(t_{1}, x\left(t_{1}\right), y\left(t_{1}\right)\right)\right.\right. \\
& =\int_{t_{1}}^{t_{N}+\frac{\varepsilon_{0}}{2 M}} \dot{U}(s, x(s), y(s)) d s \leq-\int_{t_{1}}^{t_{N}+\frac{\varepsilon_{0}}{2 M}} \frac{1}{e} w(x(s)) d s \leq \\
& \leq-\frac{1}{e} \cdot \sigma \cdot \sum_{J=1}^{N} \int_{t_{J}}^{t_{J+1}+\frac{\varepsilon_{0}}{2 M}} d s=-\frac{1}{e} \cdot \sigma \cdot \frac{N \varepsilon_{0}}{\overline{2} M} \text {. }
\end{aligned}
$$

Assim quando $N \rightarrow \infty$ temos $U(t) \rightarrow-\infty$ ao longo da se quência $\left\{t_{n}\right\}$ o que contradiz a relação $(4.3 .7)$ e portanto a prova se completa. 


\section{CAP ITULO 5}

\section{APLICAÇÃO}

\section{1 - INTRODUÇAO A TEORIA DO CONTROLE}

\section{1 .1 - Apresentação}

A teoria do controle é motivada no estudo de "siste mas". Intuitivamente nós podemos considerar um sistema como sendo um conjunto de componentes que se interagem, sujeito a vārias "entradas" e produzindo vārias "saídas".

Os tipos de sistemas com os quais estamos mais fami liarizados são os sistemas mecânicos, como os relógios; siste mas mecânico-quỉmicos, como os automóveis; sistemas biológicos, tais como o próprio corpo humano; e muitos outros. Estes sim ples exemplos são suficientes para mostrar a relevância do con ceito de "sistemas".

\section{1 .2 - Esquematização}

Erequente indicar cada intuição e exposição do con ceito de um sistema em termos de um diagrama de bloco.

Uma primeira tentativa é dada pelo esboço:

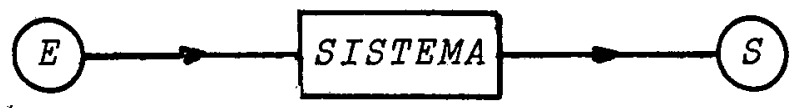

Este è o caso mais simples de um diagrama. Em geral - sistema pode estar sujeito a uma mais complexa variedade de entradas e saídas.

5.1.3 - Aperfeiçoamento do comportamento de Sistemas

Uma grande parte dos sistemas não possue um comportą 
mento regular. De fato; è comum observar-se a existência de sistemas que não operam de forma desejāvel.

E o caso, por exemplo, dos sistemas econômicos, sujeí tos a inflação e depressão; dos sistemas humanos sujeitos a doenças, como o câncer, moléstias do coração, etc.

Existem vários modos de melhorar o comportamento de um sistema. Podemos escolher entre a construção de um novo sistema ou a mudança de suas entradas.

Em alguns casos, como sabemos, a solução ideal é dada por um sistema novo. Este é o caso do sistema químico-mecâni co dado por um carro superado.

Em outros casos, como por exemplo quando tratamos com sistemas humanos; foge da nossa alçada uma solução semelhante à citada anteriormente.

Consequentemente nós podemos pensar em termos de um programa mais praticável de alteração do objetivo do sistema, ou então, modificando as entradas.

Um modo de realizar, isto é, observar a saída do sis tema, e reformular adequadamente o esquema de acordo com o des vio da atual saída, e a saída almejada que comumente è chamada um modelo para o sistema.

A idéia básica do "feedback" controlado é esquematiza da abaixo.

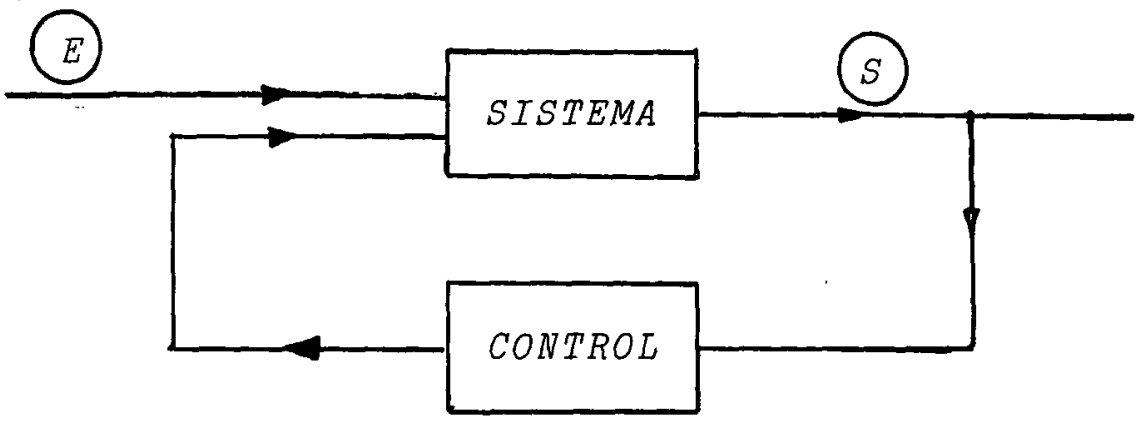


Naturalmente existe uma forma de realizar tais altera ções, de modo operacional que não nos é revelada pelo simples esquema dado acima.

Nos modelos matemāticos em geral; entre os quais se enquadra a aplicação do nosso trabalho, é conveniente analisar a situação de forma mais acurada. Por exemplo a operação de observar a saída em geral è completamente distinta da operação de exercer o controle.

Por conseguinte um esquema um pouco mais detalhado po de ser apresentado, se levarmos em consideração que em geral, observamos o sistema em si mesmo, bem como a sua saida; e que - trabalho de decisão concernente a escolha de uma ação de con trole è realmente uma operação distinta daquela de mostrar a ação de controle decidida. Tal comportamento justifica o dia grama abaixo.

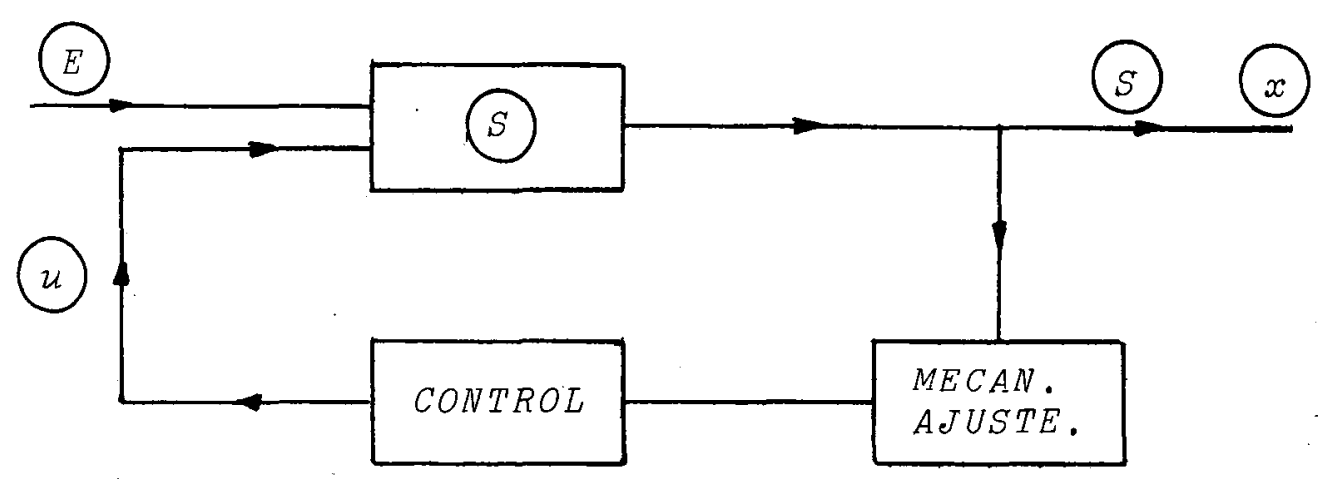

\subsection{4 - O Modelo Matemātico}

o modelo matemático para o estudo de estabilidade em controle requer que o sistema a ser controlado seja descrito por um sistema de equações diferenciais dado por $\dot{x}_{i}=x_{i}\left(t, x_{1}\right.$, $x_{2}, x_{3}, \ldots, x_{n}, u_{1}, \ldots, u_{n}$, onde $\dot{x}_{i}=\frac{d x_{i}}{d t}, i=1,2, \ldots, n$.

$\mathrm{Na}$ forma vetorial ele vem apresentado como $\bar{x}=X(t, x, u)$.

o objetivo é selecionar o controle u de modo que osis 
tema seja mantido próximo de algum estado desejado. E usual escolher como estado desejado a origem $x=0$ (erro zero). No caso geral o controle u será uma função do estado $x$ do sistema e do tempo; com a seleção de um controle especifí co o sistema acima se transforma em:

$$
\dot{x}=x(t, x, u(x, t))=f(t, x)
$$

Recaímos então no modelo de sistema abordado no inî cio destas notas.

Se a planta, ou sistema em controle, estiver sujeita a perturbações e variações do ambiente, então é melhor ter o controle adaptável, fazendo com que o estado desejado compor te-se cada vez mais como um estado de equilíbrio estável ou, melhor ainda, como um estado assintoticamente estável.

Nesta operação desempenha papel importante o mecanis mo de ajuste assinalado no esquema (3) do último parágrafo.

\section{2 - UM PROBLEMA DE ESTABILIDADE EM CONTROLE}

Este exemplo esclarece que se tivermos informações su彑 ficientes, as quais podem ser de origem estatística, sobre fun ções desconhecidas na planta a ser controlada, então o contro le adaptável é possível. Faremos uso essencialmente do Teore $\operatorname{ma} 4.3$.

Nesta aplicação o estado $x$ representa o erro e $y$ está relacionado com certos parâmetros a serem controlados.

o que se quer, entre outras coisas, é aquilo que o Teorema 4.3 propõe, is to é; que depois do sistema ter sido ope rado por um determinado intervalo de tempo, o erro $x$ tenda a zero e os parâmetros sob controle permaneçam limitados. 
A forma geral do sistema de controle adaptável, como vimos no esquema (3) do parágrafo 5.1 .3 é dada pelo diagrama abaixo.

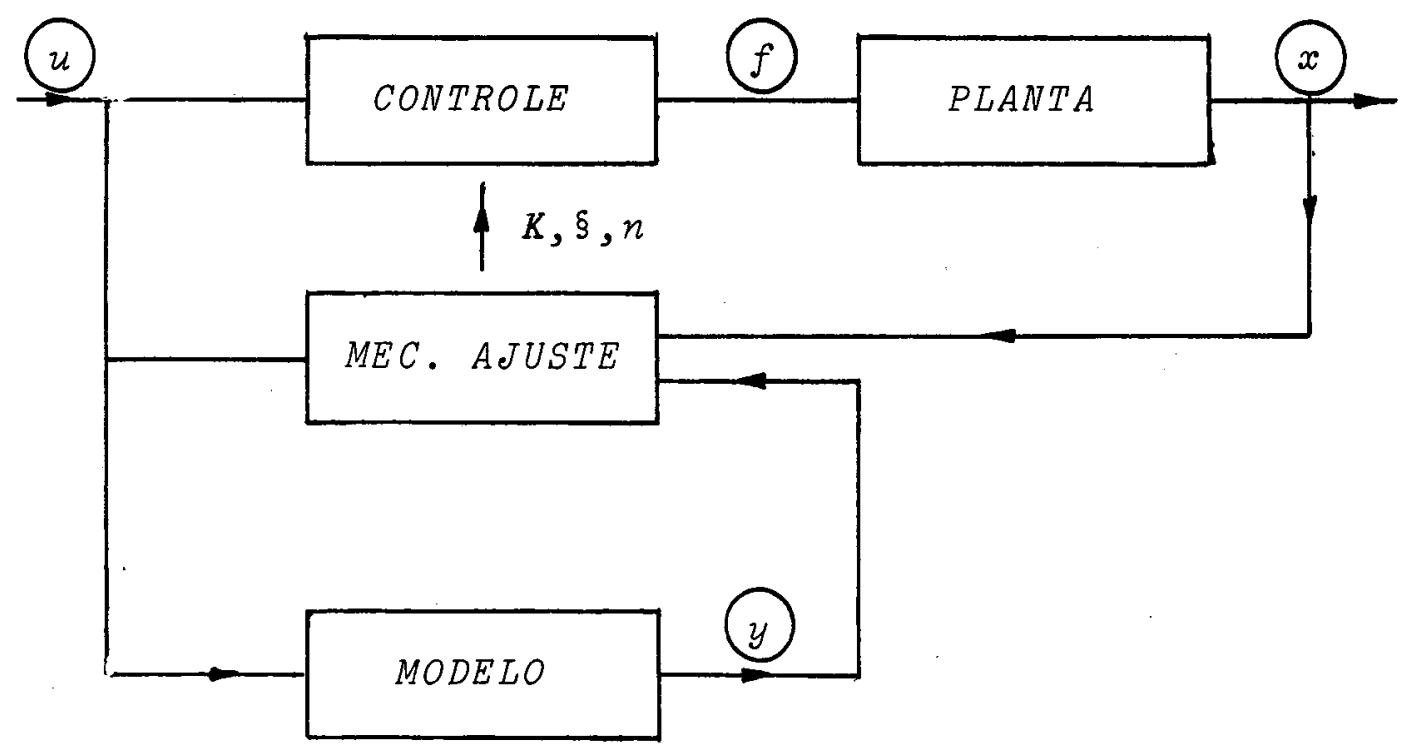

O sistema associado ao diagrama é dado por:

$$
\left[\begin{array}{l}
\dot{x}=\alpha(t) A x+\beta(t) p(t, x)+f \rightarrow \text { pranta } \\
\dot{y}=A y+u(t) \rightarrow \text { modezo }
\end{array}\right.
$$

Os estados $x$ e $y$ são vetores do $\mathbb{R}^{n}$. A matriz $A$ de or dem $n$ admịte valores característicos com parte real negativa, e $u(t)$ é contínua e limitada pari $t \geq 0$. Para a planta é as sumido que $p(t, x)$ seja conhecida e limitada para $t \geq 0$ e todo $x$.

Agora, tudo aquilo que é conhecido sobre as funções escalares $\alpha(t)$ e $\beta(t)$ é que são contínuas e tem derivadas limi tadas com $\alpha(t) \rightarrow \alpha_{0}$ e $\beta(t) \rightarrow \beta_{0}$ para $t \rightarrow \infty$.

Não é assumido que $\alpha_{0}$ e $\beta_{0}$ sejam conhecidos. A ques tão que se põe então è: "Determinar $f$ tal que a planta siga o modelo $(\varepsilon(t)=x(t)-y(t) \rightarrow 0$ quando $t \rightarrow \infty)$ e os parâmetros de controle permaneçam limitados." 
Existem razões heurIsticas para tomar $f$ da forma:

$f=K[(1-\xi) A x-n p(t, x)+z]$

onde $K, \xi$ e $\eta$ satisfazem ao sistema:

$$
\begin{aligned}
& \| \dot{K}=g_{1}(t, x, y, K, \xi, n) \\
& \| \dot{\xi}=g_{2}(t, x, y, K, \xi, n) \\
& \| \dot{\eta}=g_{3}(t, x, y, K, \xi, n)
\end{aligned}
$$

E claro que a fim de obtermos $f$ precisamos conhecer as funções $g_{1}, g_{2}$ e $g_{3}$.

$\operatorname{Com} \varepsilon=x-y$ a equação diferencial de $\varepsilon$ fica:

$$
\begin{aligned}
\dot{\varepsilon}=A \varepsilon & +\left(\alpha_{0}-\xi\right) A x+\left(\beta_{0}-\eta\right) p+(K-1) K^{-1} f \\
& +\left(\alpha-\alpha_{0}\right) A x+\left(\beta-\beta_{0}\right) p
\end{aligned}
$$

Por hipōtese A satisfaz $\operatorname{Re}\left(\lambda_{J}\right)<0 \quad J e\{1,2, \ldots, n\}$. Logo, para cada matriz $C$ definida positiva, existe $Q$ definida positiva tal que $A^{\prime} Q+Q A=-2 C$, onde $A^{\prime}$ é a transposta de A. $[5]$

Selecionamos $C$ qualquer e consequentemente $Q$ e defini mos

$$
2 v=\varepsilon^{\prime} Q \varepsilon+c_{1}(K-1)^{2}+c_{2}\left(\xi-\alpha_{0}\right)^{2}+c_{3}\left(n-\beta_{0}\right)^{2}(5.2 .3)
$$

onde $c_{1}, c_{2}, c_{3}$ são constantes positivas.

Observamos inicialmente que pondo $z=\left(z_{1}, z_{2}, z_{3}\right)$ onde $z_{1}=K-1, z_{2}=\xi-\alpha_{0} e z_{3}=n-\beta_{0}$, o ponto $(0,0)$ do siste ma $(\varepsilon, z)$ governados pelas equações $(5.2 .2)$ e $(5.2 .1)$ respect vamente, è dado por $\varepsilon=0, \xi=\alpha_{0}, K=1, \eta=\beta_{0}$.

Segundo esta notação fica evidente que $v(0,0)=0$, $v(\varepsilon, z)$ è definida positiva e possue derivadas parciais contí nuas em relação a $\varepsilon$ e $z$, verificando-se assim o Teorema 4.3 em seu item (a). 
o Teorema está sendo aplicado tomando $p=n$ e $q=3$, além disso os papeis de $x$ e $y$ no teorema serão aqui representa dos por $\varepsilon$ e $z$ respectivamente.

Do modo como foi definido o funcional $v$ o item (b) es tá automaticamente verificado.

A exigência dada pelo item (d) do Teorema 4.3 também é satisfeita se lembrarmos que na expressão (5.2.2) $x=\varepsilon+y$ e que o modelo em qualquer estágio do controle é sempre conhe cido.

Calculando a derivada $\dot{v}$ encontramos :

$$
\begin{aligned}
\dot{v}= & -\varepsilon^{\prime} C \varepsilon+(K-1)\left(c_{1} g_{1}+K^{-1} f^{\prime} Q \varepsilon\right)+ \\
& +\left(\xi-\alpha_{0}\right)\left(c_{2} g_{2}+\varepsilon^{\prime} C \varepsilon-y^{\prime} A^{\prime} Q \varepsilon\right)+ \\
& +\left(n-\beta_{0}\right)\left(c_{3^{g}} g-p^{\prime} Q \varepsilon\right)+ \\
& +\left(\alpha-\alpha_{0}\right)\left(y^{\prime} A^{\prime} Q \varepsilon-\varepsilon^{\prime} C \varepsilon\right)+ \\
& +\left(\beta-\beta_{0}\right) p^{\prime} Q \varepsilon .
\end{aligned}
$$

A seleção dos $g_{i}$ agora se torna evidente; dado que queremos que o funcional $v$ esteja nas condições do Teorema 4.3. Por conseguinte pomos:

$$
\begin{aligned}
& \| \cdot g_{1}=-c_{i}^{-1} K^{-1} f^{\prime} Q \varepsilon \\
& \| \cdot g_{2}=-c_{2}^{-1}\left(\varepsilon^{\prime} C \varepsilon-y^{\prime} A^{\prime} Q \varepsilon\right) \\
& \| \cdot g_{3}=+c_{3}^{-1} p^{\prime} Q^{\prime} \varepsilon
\end{aligned}
$$

e obtemos assim $\dot{v}$ dado por:

$\dot{v}=-\varepsilon^{\prime} C \varepsilon+\left(\alpha-\alpha_{0}\right)\left(y^{\prime} A^{\prime} Q \varepsilon-\varepsilon^{\prime} C \varepsilon\right)+\left(\beta-\beta_{0} p^{\prime} Q \varepsilon\right.$.

A fim de completarmos a verificação que o funcional $v(\varepsilon, z)$ satisfaz às exigências do Teorema 4.3 resta conferir o item $(c)$. 
Da relação anterior temos:

$$
\begin{aligned}
\dot{v}=-\varepsilon^{\prime} C \varepsilon & +\left(\alpha-\alpha_{0}\right)^{\prime} y^{\prime} A^{\prime} Q \varepsilon-\left(\alpha-\alpha_{0}\right) \varepsilon^{\prime} C \varepsilon+ \\
& +\left(\beta-\beta_{0}\right) p^{\prime} Q \varepsilon .
\end{aligned}
$$

Logo, escolhendo $w(\varepsilon)=\varepsilon^{\prime} C \varepsilon \rightarrow w$ é definida positi

va.

Agora se $\bar{h}_{1}(t)=\alpha(t)-\alpha_{0}$; sendo que $\alpha \rightarrow \alpha_{0}$ por hipó tese, decorre que $\bar{h}_{1} \in M_{0} \rightarrow h_{1}=\bar{h}_{1} y^{\prime} A^{\prime} Q \in M_{0}$ pois de acordo com as hipóteses temos ||$y^{\prime} A^{\prime} Q||$ é uma parcela limitada.

Se $q_{1}(\varepsilon, z)=\varepsilon$ a segunda parcela de $\dot{v}$ é do tipo $h_{1}(t) \cdot q_{1}(\varepsilon, z)$ com $h_{1} \in M_{0}$ e $q_{1}(\varepsilon, z)$ contínua no par $(\varepsilon, z)$.

Tomando $h_{2}(t)=-\left(\alpha(t)-\alpha_{0}\right) \rightarrow h_{2} \in M_{0}$. De modo anā logo se $q_{2}(\varepsilon, z)=\varepsilon^{\prime} C \varepsilon \rightarrow q_{2}(\varepsilon, z)$ é contínua no par $(\varepsilon, z)$.

A última parcela de $\dot{v}$ se comporta de forma anāloga às anteriores recordando-se que $p(t, x)$ é limitada para $t \in J$ e $x \in I R^{n}$.

Assim $\quad \dot{v}=-w(\varepsilon)+h_{1}(t) q_{1}(\varepsilon, z)+h_{2}(t) q_{2}(\varepsilon, z)+$ $+h_{3}(t) q_{3}(\varepsilon, z)$ com $w(\varepsilon)$ def. positiva, $h_{i} \in M_{0}$ e $q_{i}(\varepsilon, z)$ con tínua no par $(\varepsilon, z) \quad i=1,2,3$.

Desta forma o funcional $v$ definido pela expressão (5.2.3) satisfaz as hipóteses do Teorema 4.3 e consequentemen te $\varepsilon=0, \quad K=1, \quad \xi=\alpha_{0}, \quad \eta=\beta_{0} \bar{e}$ eventualmente uniformemen te estável e para cada $r>0$, existe $T_{0}>0$ tal que

$$
\begin{aligned}
\left\|\varepsilon\left(t_{0}\right)\right\|^{2} & +\left(K\left(t_{0}\right)-1\right)^{2}+\left(\xi\left(t_{0}\right)-\alpha_{0}\right)^{2}+\left(n\left(t_{0}\right)-\right. \\
& \left.-\beta_{0}\right)^{2}<r^{2}
\end{aligned}
$$

para qualquer $t_{0} \geq T_{0} \rightarrow \varepsilon(t) \rightarrow 0$ e as funções $K(t), \quad \xi(t)$, $\eta(t)$ são limitadas para $t \geq t_{0}$.

E claro que a conclusão supera as nossas expectativas, 
pois ela nos diz que, no tempo, a região na qual um comporta mento satisfatório é obtido torna-se o espaço inteiro.

Não possuindo maiores informações sobre as funções des conhecidas que descrevem a planta em controle, tal resultado talvez é o melhor que possa ser obtido.

Em particular, se $\alpha \equiv \alpha_{0}$ e $\beta \equiv \beta_{0}$ então todas as solu ções $\varepsilon(t), K(t), \xi(t)$ e $\eta(t)$ são limitadas e cada $\varepsilon(t) \rightarrow 0$ quan $t \rightarrow \infty$. 



\section{APENDICE}

\section{DEFINIÇסES}

1 - v: $\Omega \mathrm{C} \mathbb{R}^{n} \rightarrow \mathbb{R}, \Omega$ aberto, é dita definida positiva se satisfaz as condições:

(a) $v(x)$ é continua, bem como suas derivadas parciais.

(b) $v(0)=0$.

(c) Para todo $x \in \Omega, x \neq 0$ temos $v(x)>0$.

2 - $v: J \times \Omega \rightarrow I R, \Omega$ aberto, $J=I R+$ é dita definida posití va se satisfaz as condições:

(a) $v(t, 0)=0$.

(b) $v(t, x) \geq w(x)$ para todo $t \in J$ e para todo $x \in \Omega$; onde $w(x)$ satisfaz a definição (1).

$3-\phi:[0, \rho) \rightarrow \mathbb{R}$ + pertence $\bar{a}$ classe $K$ se satisfaz as condi ções :

(a) $\phi(0)=0$.

(b) фè contínua.

(c) ф é monótona estritamente crescente.

Teorema:

Dado o sistema

$\dot{x}=f(t, x)$

se $f(t, 0)=0, f(t, x)$ è localmente Lipschitziana em $x$ uniforme mente em $t$ e a solução $x=0$ de (I) é uniformemente assintotí 
camente estävel, logo existe $r_{1}>0, K=K\left(r_{1}\right)>0$, uma função definida positiva $b(r)$, uma função positiva $c(r)$, ambas defini das para $r \in\left[0, x_{1}\right]$; e uma função escalar $v(t, x)$ definida $e$ continua para $t \geq 0, \quad x \in \mathbb{R}^{n}$ com $|x| \leq r_{1}$; tais que:

$$
\begin{aligned}
& \text { (a) }|x| \leq v(t, x) \leq b(|x|) \\
& \text { (b) } \quad \dot{v}(t, x) \leq-c(|x|) v(t, x) \leq-|x| c(|x|) \\
& \text { (c) }|v(t, x)-v(t, y)| \leq K|x-y|
\end{aligned}
$$

para todo $t \geq 0, x, y$ no $\mathbb{R}^{n}$ com $|x| \leq r_{1},|y| \leq r_{1}$.

D. - Vide referência bibliogrăfica [3].

Lema:

Supomos $f$ nas condições do teorema precedente, $v$ è a função dada no teorema, $g: J \times I R^{n} \rightarrow I R^{n}$ è uma função qual quer, e consideremos o sistema perturbado

$$
\dot{x}=f(t, x)+g(t, x) .
$$

Logo, se verifica a desigualdade:

$$
\dot{v}_{(I I)}(t, x) \leq-c(|x|) v(t, x)+K|g(t, x)| \text {. }
$$

D. - Referência bibliográfica [3]. 


\section{BIBLIOGRAFIA}

[1] BELMAN, RICHARD (1971). Introduction to the Mathematical Theory of Control Process. A. Press, New York.

[2] HALANAY, A. (1966). Differential Equations Oscillation Time Lags. A. Press, New York.

[3] HALE, JACK (1969): Ordinary Difberential Equations. Wiley, New York.

[4] LAKSMIKANTHAM, V.; LEELA, S. (1961). Differential and Integral Inequalities. A. Press, New York.

[5] LA SALLE - LEFSCHETZ (1961). Stability by Liapunov's Di rect Method. A. Press, New York.

[6] ONUCHIC, NELSON (1971). Equações Diferenciais com Retar damento no Tempo. 88 Colóquio, Poços de Caldas - MG.

[7] ONUCHIC, NELSON (1970). Notas de Aula de um Curso de Equações com Retardamento no Tempo - Estabilidade Segun do Lyapunov. Escola de Engenharia de são carlos - USP.

[8] STRAUSS, A. and YORKE, JAMES A. (1967). Perturbation Theo rems for ordinary Differential Equations. Reprinted from JOURNAL of Differential Equations. 